\title{
LA CHIMIE DES PLASMAS ET SES DÉbOUCHÉS A COURT TERME SUR DES SYNTHĖSES INORGANIQUES A CARACTÈRE INDUSTRIEL
}

\author{
P. FAUCHAIS et E. BOURDIN \\ Laboratoire de Thermodynamique, U.E.R. des Sciences \\ Université de Limoges, France
}

\begin{abstract}
Résumé. - La première partie de cet article est consacrée à une description essentiellement qualitative des diverses connaissances fondamentales, empiriques et semi-empiriques que l'on possède actuellement dans le domaine de la Chimie des Plasmas. Compte tenu de l'étendue considérable de ce sujet, nous nous sommes souvent restreints au cas des plasmas thermiques, en particulier dans la seconde partie où nous présentons une série d'exemples d'application qui ont été développés dans le secteur industriel. Ces exemples couvrent aussi bien le cas des réactions gaz-gaz dans un arc électrique (synthèse de l'acétylène) que celui des réactions gaz-solides (traitement de zircon pour l'obtention de zircone, fours d'extraction de minerais de fer ou de ferroalliages). Un accent particulier a été mis sur l'importance des études économiques de ces techniques de pointe.
\end{abstract}

Abstract. - The first part of this paper is devoted to qualitative description of the different fundamental, empirical and semi-empirical knowledges that one have nowadays in the field of Plasma Chemistry. On account of the extend of this subject we have often been constrained to restrict these considerations to thermal plasmas, in particular in the second part devoted to some examples coming from industrial state. These examples deals with the case of gas-gas reactions in electric arcs (acetylene synthesis), and with the case of solid-gas reactions (treatment of zircon $\mathrm{ZrSiO}_{4}$ for the obtainment of zirconia $\mathrm{ZrO}_{2}$, extractive metallurgy with iron or ironalloys ores). A particular effort has been pointed on the economical aspect of these up to date technics.

1. Introduction. - La diminution rapide des réserves mondiales d'hydrocarbures ou de gaz naturels et l'augmentation considérable de leur prix impliquent une réévaluation complète de leur utilisation respective en tant que source d'énergie. Compte tenu des réserves dont on dispose en énergies diverses (cf. Tableau I de [1]), le développement de l'utilisation de l'électricité produite soit à partir des combustibles nucléaires, soit à partir des réserves de charbon semble être une solution logique au problème énergétique à long terme et ce tout particulièrement en France où d'ici 1985 l'E.D.F. compte doubler sa production (Fig. 1). Ce nouvel échantillonnage des divers types d'énergie nécessite naturellement en retour que de nombreux processus chimiques à hautes températures utilisent des dispositifs de chauffage à énergie électrique. C'est pourquoi les générateurs de plasma à arc électrique présentent un intérêt de plus en plus grand, d'autant qu'ils peuvent être appliqués à une gamme très étendue de processus à hautes températures.

$\mathrm{Au}$ cours des dernières décades, de nombreuses recherches à caractère technologique ont été lancées en chimie des plasmas mais seuls quelques rares processus ont débouché à grande échelle sur le plan industriel. Une des principales raisons de cette non-commercialisation semble être le coût élevé de l'électricité, situa-

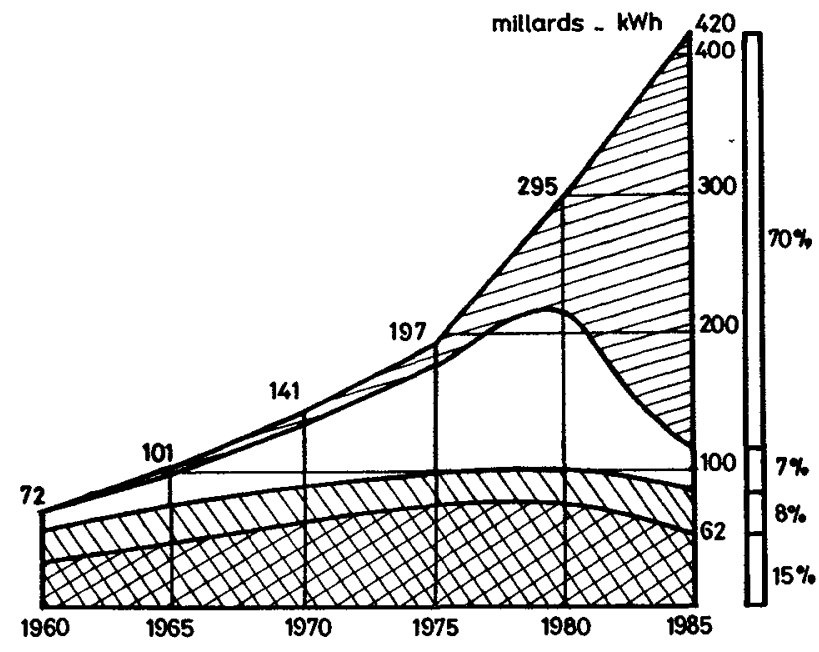

Fig. 1. - Evolution de la production nationale d'électricité de 1960 à 1985. Parts des diverses énergies primaires : nucléaire, $\square$ combustible importé, $\square$ combustible national, hydraulique.

tion qui est en pleine évolution puisque, dans le futur, l'électricité sera certainement la source d'énergie la plus stable en terme de disponibilité et de coût. C'est pourquoi nous nous proposons, dans cet article, 
TABLEAU I

Sources mondiales d'énergie $\left(10^{18} \mathrm{~K}\right.$.Joules)

Houille
Pétrole
Gaz
Uranium (fission)
Surrégénérateurs

Fusion

après avoir rappelé les problèmes posés par la chimie des plasmas, de développer quelques exemples de processus susceptibles de déboucher à moyen terme dans le secteur industriel. Nous nous limiterons pour ceci aux dispositifs utilisant les arcs électriques de grande puissance susceptibles de traiter plusieurs tonnes de matériaux par jour et, ne parlerons pas, au niveau des applications, des processus basse pression pourtant déjà utilisés industriellement pour produire de petites quantités de matériaux très purs.

2. Bref historique. - Si l'on excepte les travaux de laboratoire de Henry et Dalton portant en 1797 sur la synthèse de l'acétylène, ceux de Buff et Hoffmann et de Berthelot entre 1860 et 1880 , le procédé industriel de Birkeland-Eyde de synthèse de l'oxyde d'azote [2] dans un arc électrique est un des premiers travaux que l'on peut classer dans le domaine de la chimie des plasmas. A la même époque (fin du XIx siècle) Moissan, Hare, Curtis [3, 4, 5] et d'autres, dans des conditions identiques, ont effectué des réactions en phase solide, ainsi que des synthèses endothermiques en phase gazeuse. Mais ces travaux très empiriques tombèrent plus ou moins dans l'oubli car on ne disposait pas d'une information suffisante sur les arcs électriques et le milieu (plasma) dans lequel ils se développent.

Plusieurs dizaines d'années plus tard, l'avènement des techniques de soudage de l'aluminium en atmosphère inerte a entraîné l'apparition des torches à plasma utilisables au laboratoire (1954). Le nombre des études publiées dans ce domaine s'est alors considérablement multiplié ; tant sur les arcs électriques [6], que sur la génération des plasmas thermiques [7-9]. L'investigation des possibilités offertes par ces plasmas pour le soudage, le découpage, les projections et la chimie [10] pouvait alors débuter, et de nombreux laboratoires aussi bien en France qu'à l'étranger, ont axé leurs recherches sur ces techniques.

Parallèlement se sont développées d'une part les recherches sur les décharges haute fréquence $[11,12]$ qui ont l'avantage d'exclure pratiquement la pollution du plasma par les électrodes et d'autre part sur un plan plus fondamental pour la compréhension des réactions, l'étude des décharges luminescentes [13].

Le tableau II résume une partie des études qui ont été publiées de 65 à 72 . L'intérêt croissant porté à ces

\section{TABLEaU II}

1904

1909

1949

1965

1961

1962

1960-1964

1956

1962

1964

1967

1968

1968

1960

1964

1966

1968

1966 à 1972
Synthèse de NO : Procédé « Birckeland Eyde » [2]. Synthèse de l'hydrazine $\mathrm{N}_{2} \mathrm{H}_{4}$ (décharge dans $\mathrm{NH}_{3}$ ) [14].

Synthèse de l'hydrazine $\mathrm{N}_{2} \mathrm{H}_{4}[15,16]$.

Synthèse de NO (plasma d'azote + oxygène) [17].

Synthèse de l'acide cyanhydrique HCN (plasma d'azote produit avec des électrodes de graphite $+\mathrm{H}_{2}$ ) [18-20].

Synthèse de $\mathrm{C}_{2} \mathrm{~N}_{2}$ (plasma d'azote produit avec des électrodes de graphite) [21].

Etudes de la combinaison $\mathrm{C}-\mathrm{F}\left(\mathrm{C}_{2} \mathrm{~F}_{4}, \mathrm{CF}_{4}\right.$ et $\mathrm{C}_{2} \mathrm{~F}_{6}$, $\mathrm{C}_{3} \mathrm{~F}_{8}$ ) [22-26].

Etudes de la combinaison $\mathrm{NF}$ [27-29] $\left(\mathrm{NF}_{3}, \mathrm{~N}_{2} \mathrm{~F}_{4}\right.$, $\mathrm{N}_{2} \mathrm{~F}_{2}$ ).

Obtention d'oxydes très finement divisés à l'aide d'un plasma d'argon + oxygène et de poudres de métal injectées dans le plasma [30-32].

Réduction d'oxydes et des minerais oxydés
de plasmas d'argon + hydrogène [33-36].

Synthèse de Carbure d'Uranium UC (électrodes en $\mathrm{UO}_{2}$ et carbone, plasma d'argon) [37].

Synthèse du Carbure de Bore $\mathrm{B}_{4} \mathrm{C}$ (introduction du bore en suspension dans l'argon + méthane) [38]. Synthèse de Carbure de Zirconium $\mathrm{ZrC}$ (par réduction de $\mathrm{ZrO}_{2}$ en présence de benzène dans un plasma $\mathrm{Ar}$ ) [39-40].

Synthèse des carbures $\mathrm{Ta}_{2} \mathrm{C}$ et $\mathrm{W}_{2} \mathrm{C}$ (par action du méthane sur les poudres de Ta et $\mathrm{W}$ dans un plasma Ar) [36, 41, 42].

Synthèse des carbures de $\mathrm{Si}$, de $\mathrm{Ti}$, de $\mathrm{Ta}$ et de $\mathrm{V}$ (par réduction des oxydes mélangés à du carbone dans un plasma d'argon) [43].

Synthèse de $\mathrm{TiN}$ et $\mathrm{Mg}_{3} \mathrm{~N}_{2}$ (plasma $\mathrm{N}_{2}+$ poudres) [44].

Synthèse de AlN et de l'oxynitrure d'Aluminium [39]. Synthèse de TiN (à partir de $\mathrm{TiCl}_{4}$ dans un plasma $\mathrm{H}_{2}+\mathrm{N}_{2}$ ) [45-48].

Synthèse de AlN (à partir de $\mathrm{AlCl}_{3}$ ) [49].

Synthèse de $\mathrm{TaN}, \mathrm{Mo}_{2} \mathrm{~N}, \mathrm{~W}_{2} \mathrm{~N}$, TiN, AlN, $\mathrm{ZrN}$, $\mathrm{TiN}, \mathrm{HgN}, \mathrm{Si}_{3} \mathrm{~N}_{4}$ (par action d'un jet de plasma d'azote sur le métal en arc transféré) [50-60].

techniques est probablement dû à une plus grande compréhension des phénomènes fondamentaux et à la possibilité de produire en laboratoire des plasmas stables et reproductibles.

3. Les plasmas. - - 3.1 DéfintTION DU PLASMA. Par définition le plasma est un milieu constitué de molécules et d'atomes, le plus souvent dans des états excités, d'ions et d'électrons, l'ensemble étant électriquement neutre.

Une des classifications possibles des divers milieux plasma repose sur leur degré d'équilibre. On trouve ainsi les plasmas

- en équilibre thermodynamique complet (CTE plasma dans la littérature anglaise),

- en équilibre thermodynamique local (LTE),

- en équilibre thermodynamique local partiel (PLTE),

- et enfin les plasmas en déséquilibre. 
La connaissance du degré d'équilibre n'a pas qu'un seul intérêt fondamental, elle est utile au chimiste du plasma pour déterminer théoriquement la composition, et expliquer avec le maximum d'exactitude les divers phénomènes cinétiques.

Dans les articles de chimie des plasmas [61-63] qui prennent en considération ces problèmes d'équilibre, la terminologie est généralement un peu différente de celle que nous venons de rencontrer. En effet, on parle de plasmas thermiques ou chauds pour les plasmas en LTE et de plasmas non thermiques ou froids pour les plasmas en non LTE. On trouve généralement dans la littérature [61-64] la distinction entre les plasmas basse et haute température; la chimie des plasmas basse température travaille dans la bande des énergies inférieures à $10 \mathrm{eV} /$ particules $\left(T<10^{5} \mathrm{~K}\right)$, tandis que celle des plasmas haute température se situe au-delà de $10^{5} \mathrm{~K}$ (ou de $10 \mathrm{eV} /$ particules) et concerne essentiellement les réactions nucléaires.

3.2 Classification SOMMaire DES Plasmas DE LABORATOIRE. - De très nombreux types de plasmas, aux propriétés physiques sensiblement différentes, produits en général par décharge électrique, ont été décrits dans la littérature. Leur état est essentiellement caractérisé par l'énergie des électrons $\left(k T_{\mathrm{e}}\right)$ et leur densité $\left(n_{\mathrm{e}}\right)$. La figure 2 [65] situe dans l'espace $\left(n_{\mathrm{e}}\right.$, $k T_{\mathrm{e}}$ ) un grand nombre de plasmas naturels et de laboratoires. L'indication de la longueur de Debye $\lambda_{\mathrm{D}}$ donne une idée de l'ordre de grandeur du volume minimal à considérer pour que l'on puisse réaliser la neutralité électrique.

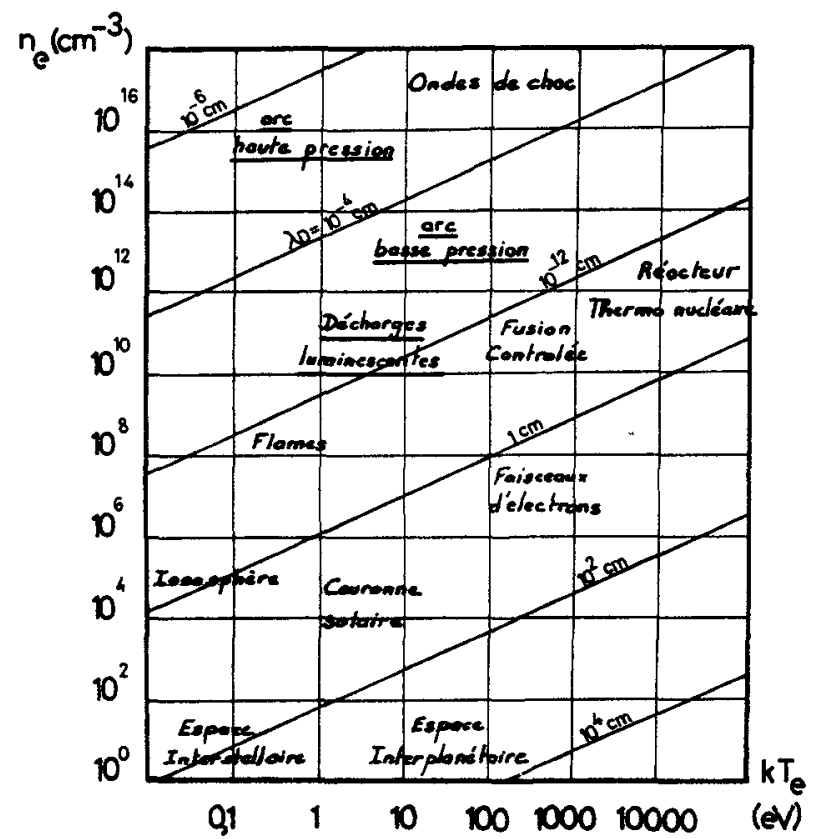

FIG. 2. - Différents plasmas caractérisés par l'énergie et la densité des électrons.

Les deux régions qui présentent le plus grand intérêt pour la chimie des plasmas sont celles des décharges luminescentes et des arcs. Les plasmas de décharge luminescente ( $p \leqslant 100$ torrs) sont entre autres caractérisés par un rapport entre la température des électrons $T_{\mathrm{e}}$ et celle du gaz $T_{\mathrm{g}}$ (particules lourdes) compris entre 10 et $10^{2}$. Cette absence d'équilibre permet d'obtenir un plasma dans lequel la température des gaz peut être voisine de l'ambiante alors que les électrons sont suffisamment énergétiques pour entrâ̂ner la rupture des liaisons moléculaires par exemple. Cette propriété rend ce type de décharge parfaitement adaptée aux réactions chimiques entre matériaux très sensibles aux effets de température (composés organiques par exemple). Les arcs à haute pression ( 1 atmosphère et au-delà) et forte intensité par contre correspondent à une situation voisine de l'équilibre $\left(T_{\mathrm{e}}\right.$ et $T_{\mathrm{g}}$ sont presque identiques [66] Figs. 3 et 4), et les températures élevées que l'on y rencontre $\left(T>4 \times 10^{3} \mathrm{~K}\right)$ rendent ce type de plasma adapté au traitement des matériaux non organiques : synthèses de réfractaires, projection et fusion, synthèses de petites molécules telles que l'acétylène, l'acide cyanhydrique ou les oxydes d'azote.

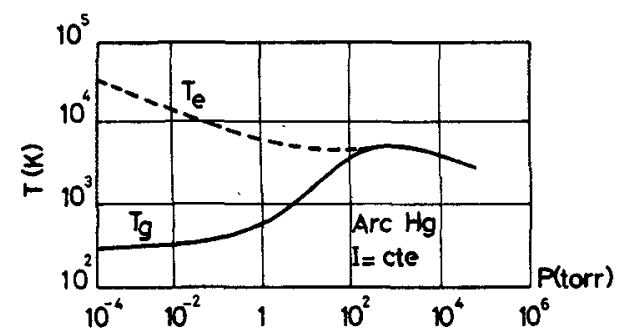

Fig. 3. - Température du gaz et des électrons en fonction de la pression.

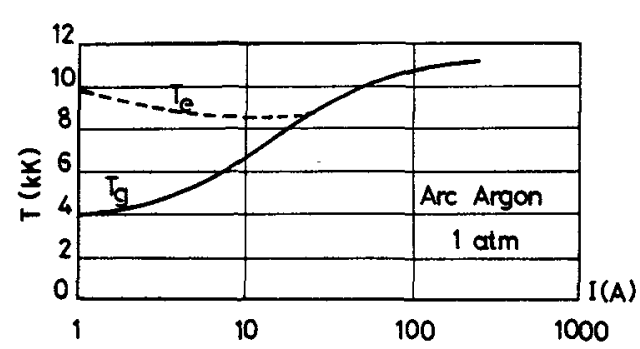

FIG. 4. - Température du gaz et des électrons en fonction de l'intensité du courant d'arc.

4. Production des plasmas. - On est souvent conduit à faire la distinction entre plasma thermique et plasma froid essentiellement sous l'aspect technologique, sans entrer dans le détail de leur définition physique exacte [67], [68].

Les décharges haute fréquence ou micro-ondes sont très souvent utilisées en chimie des plasmas car elles permettent d'obtenir des plasmas non contaminés par les électrodes. Le couplage de la puissance haute fréquence avec le gaz est réalisé par capacité ou self en radio-fréquence, par guide d'ondes et cavité résonnante en micro-ondes [69] à [72]. Ces décharges fonctionnent généralement entre 0,1 et 100 torrs et quelquefois jusqu'à une atmosphère. La température des 
électrons y est toujours beaucoup plus élevée que la température des particules neutres et on a donc là des plasmas utilisés essentiellement en chimie sélective par transfert d'excitation.

Dans le domaine de la chimie des très hautes températures on utilise des plasmas thermiques. Les chalumeaux fonctionnent la plupart du temps avec un arc électrique soufflé, le gaz étant essentiellement chauffé par effet Joule. La stabilisation de l'arc peut être assurée par un champ magnétique [73-76, 1], par une stabilisation aérodynamique [77-79] ou par les parois [80-86].

Pour augmenter la puissance dissipée dans les colonnes de plasma produit par arcs électriques, on peut utiliser :

a) des écoulements à arcs transférés pour lesquels l'une des électrodes ne sert qu'à créer un canal de guidage et' à stabiliser l'arc (en général le pôle positif est transféré à une électrode externe au générateur [87]),

b) des écoulements à arcs superposés où une puissance importante est superposée aux écoulements plasma de deux ou plusieurs générateurs [88, 89]. Cette superposition peut d'ailleurs être utilisée en courant alternatif [90-92],

c) des écoulements à arcs superpo-transférés qui sont une association des deux systèmes précédents [93].

5. Généralités sur la chimie des plasmas. - Nous nous trouvons donc en présence d'un milieu où, suivant le mode de génération utilisée, les électrons peuvent atteindre des énergies de l'ordre de $10 \mathrm{eV}$, les particules lourdes des températures de $2 \times 10^{4} \mathrm{~K}$ et où la gamme des énergies de réactions possibles est très grande [94] (Fig. 5). Dans un tel environnement,

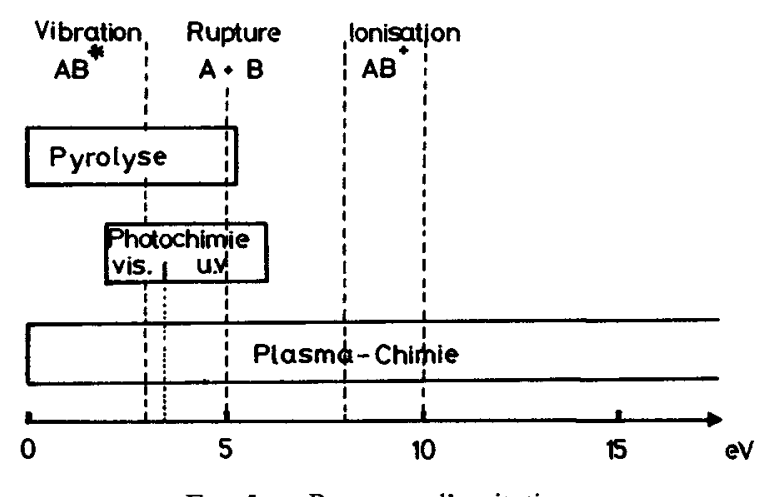

Fig. 5. - Processus d'excitation.

la chimie des plasmas est radicalement différente de la chimie traditionnelle [63]; les atomes excités sont susceptibles de réagir pour donner des produits difficiles sinon impossibles à obtenir par les moyens classiques.

Une étude très poussée de ces systèmes doit être entreprise afin de prévoir des réactions auxquelles on peut s'attendre, malheureusement on se heurte à de nombreux obstacles dont la plupart n'ont pas encore été franchis : les conditions expérimentales sont généralement difficiles à définir avec précision, la connaissance des propriétés à haute température des atomes, des molécules et de la plupart des radicaux libres (les ions négatifs par exemple) est faible et dans la majorité des cas les données cinétiques et thermodynamiques sont absentes. De plus il faut étudier les processus de transfert de l'énergie du plasma vers les réactifs, par convection gazeuse, par collision électronique, atomique et moléculaire et par rayonnement $[95,9]$.

Les réactions dépendent de nombreux paramètres et avant d'atteindre un état d'équilibre le système passe souvent par de nombreuses étapes soumises aux phénomènes cinétiques tant physiques pour les distributions d'énergie sur les différents degrés de liberté, que chimiques pour les réactions proprement dites. Naturellement, ces cinétiques physique et chimique sont intimement couplées ce qui, dans de nombreux cas, ne permet plus d'utiliser les méthodes classiques basées sur une distribution Maxwellienne de l'énergie dans le système. De plus, lorsque les méthodes classiques de la cinétique chimique sont supposées raisonnablement pouvoir être appliquées, l'étude présente de grandes difficultés du fait des grandes vitesses de réaction qui sont elles-mêmes fonction de processus physiques complexes tels que la diffusion, les transferts turbulents et le mélange des composants réactifs au niveau microscopique.

En résumé, dans le cas le plus général, il convient donc d'étudier :

- les processus élémentaires de collision,

- la thermodynamique du mélange (composition, enthalpie, énergie libre...),

- les cinétiques chimiques et physiques,

- la dynamique des gaz pour le mélange des espèces réactives,

- les transferts de chaleur et de masse,

et naturellement l'interaction de tous ces processus. Une telle tâche est donc beaucoup trop complexe et il convient, comme toujours en pareil cas, de faire un certain nombre d'hypothèses simplificatrices et de disjoindre les divers processus.

De plus la trempe des produits obtenus joue un rôle décisif notamment pour les synthèses en phase gazeuse :

- soit lorsqu'il s'agit de faire réagir des espèces excitées ou des atomes présents uniquement à très haute température mais dont les espèces de synthèse obtenues se décomposent très rapidement lors $\mathrm{du}$ refroidissement (ainsi par exemple la synthèse des oxydes de l'azote s'effectue tant à haute température que lors de la phase de trempe à partir des atomes d'azote et d'oxygène [96]),

- soit lorsque les produits à obtenir ne peuvent l'être qu'à très haute température et que, bien que stables lorsqu'ils sont isolés à la température ambiante, 
ils se décomposent aux températures intermédiaires dans le mélange. La trempe a alors pour but d'éviter leur décomposition lors du refroidissement. C'est par exemple le cas de la formation de l'acétylène à partir du méthane [97] à [99].

Pour ces deux catégories de réaction, la sélection et la mise en œuvre des processus de trempe sont déterminantes pour le rendement. Des vitesses de trempe insuffisantes ou un retard de la trempe conduisent à la quasi-disparition de l'espèce recherchée.

D'une manière générale les plasmas peuvent être utilisés en chimie [100] comme :

1) source d'énergie très concentrée c'est-à-dire source de chaleur intense à haute température,

2) source d'ions positifs et négatifs, précurseurs des réactions entre ions, molécules, états excités et électrons,

3) source lumineuse intense pour les réactions de photochimie.

La chimie des plasmas à haute température semble en particulier très prometteuse au niveau des réalisations industrielles de réactions possédant les caractéristiques suivantes :

- l'état d'équilibre est favorable à haute température,

- les vitesses de réaction sont considérablement accrues lorsque la température augmente,

- les produits sont formés dans des conditions de non-équilibre (trempe),

- les produits de base sont très bon marché et ne présentent pas des teneurs en produit final suffisantes pour être économiquement exploités par les méthodes classiques,

- les produits de base se présentent sous une forme inexploitable au moyen des dispositifs de chimie classique.

La chimie des plasmas hors d'équilibre semble elle très prometteuse :

- pour la synthèse de produits très purs tels que les semi-conducteurs [101] (utilisation de plasmas microondes et HF),

- pour la modification des propriétés de surface (composition et structure) en particulier les polymères [102] (micro-ondes, HF, décharges luminescentes),

- pour la synthèse de films minces de polymères [102, 103] (micro-ondes, HF, décharges luminescentes) ou de composés métalliques (arcs basses pressions, HF, décharges luminescentes).

6. Les calculs en chimie des plasmas. -6.1 LES RÉACTIONS ÉLÉMENTAIRES. - En l'absence d'équilibre thermodynamique l'état du plasma est défini à la fois par les paramètres externes et par les paramètres internes c'est-à-dire les constantes des réactions élémentaires (très liées à la technique de production du plasma) qui contrôlent la création et la destruction des particules. Les méthodes permettant de calculer l'état de non-équilibre [104] sont beaucoup moins nombreuses que celles dont on dispose pour l'équilibre. Notre connaissance des constantes cinétiques est très insuffisante pour un calcul quantitatif dès que l'on se situe dans le domaine des hautes pressions ( $\sim 1 \mathrm{~atm}$ ), cependant des données semi-quantitatives ou même qualitatives permettent sinon de calculer, du moins de déterminer les conditions optimales de la synthèse recherchée.

Les collisions élastiques sont les mécanismes majeurs du transfert de quantité de mouvement entre les particules, elles ont donc un rôle prépondérant dans le calcul des propriétés de transport de translation.

Les collisions inélastiques créent et détruisent les différentes espèces chimiques et états excités. Au cours de ces collisions l'échange d'énergie cinétique et interne est important, elles jouent donc un rôle considérable dans le bilan des énergies. Ces collisions inélastiques sont caractérisées par leur section efficace de collision et par un taux de réaction $k$ qui s'exprime en fonction de cette section efficace et des fonctions de distribution des vitesses [105]. Pour les collisions inélastiques à deux corps un ordre de grandeur typique de ce taux de réaction $k$ est $10^{-11} \mathrm{~cm}^{3} / \mathrm{s}$ et pour des collisions inélastiques à trois corps une valeur typique de ce taux est

$$
k=10^{-32} \mathrm{~cm}^{6} / \mathrm{s} \text { [106] . }
$$

Nous ne reprendrons pas ici la liste des principaux processus de collisions inélastiques et on pourra pour cela consulter par exemple Delcroix [106, 107]. Nous nous bornerons à rappeler que les principales réactions ont lieu soit entre particules lourdes et électrons, soit entre particules lourdes où interviennent notamment, et souvent de façon prépondérante, les états métastables [108] qui ne peuvent pratiquement se désexciter que par collisions. De très nombreux calculs et de très nombreuses expériences ont été faits pour déterminer la valeur des sections efficaces $\sigma$ de ces processus. On peut citer par exemple l'ouvrage de Gilardini [109] pour les collisions élastiques et ceux de Mac Daniel [110, 111] et de Massey [70] pour les collisions inélastiques. Un très grand nombre d'expressions de $k$ en fonction de $\sigma$ et des fonctions de distribution de vitesse $f(v)$ a été donné, tenant compte notamment de la perturbation de $f(v)$ par les interactions entre électrons et neutres $[112,113]$ et par les réactions chimiques [114]. Pour illustrer la complexité du problème, nous avons résumé, d'après Bell [115], dans le tableau III les réactions dont il convient en principe de tenir compte pour étudier une décharge basse pression dans l'oxygène.

Disposant des valeurs des coefficients $k$ on peut écrire les équations chimiques en déséquilibre, en se souvenant qu'elles ont les caractéristiques suivantes :

a) les collisions inélastiques sont très importantes, 


\section{TABleaU III}

Réactions élémentaires dans une décharge dans l'oxygène à basse pression

Réaction

Ionisation

1) $\mathrm{e}+\mathrm{O}_{2} \rightarrow \mathrm{O}_{2}^{+}+2 \mathrm{e}$

2) $\mathrm{e}+\mathrm{O} \rightarrow \mathrm{O}^{+}+2 \mathrm{e}$

Ionisation dissociative

3) $\mathrm{e}+\mathrm{O}_{2} \rightarrow \mathrm{O}^{+}+\mathrm{O}$

Attachement dissociatif

4) $\mathrm{e}+\mathrm{O}_{2} \rightarrow \mathrm{O}^{-}+\mathrm{O}$

5) $\mathrm{e}+\mathrm{O}_{2} \rightarrow \mathrm{O}^{-}+\mathrm{O}^{+}+\mathrm{e}$

Dissociation

6) $\mathrm{e}+\mathrm{O}_{2} \rightarrow 2 \mathrm{O}=\mathrm{e}$

Formation de métastable moléculaire

7) $\mathrm{e}+\mathrm{O}_{2} \rightarrow \mathrm{O}_{2}\left({ }^{1} \Delta_{\mathrm{g}}\right)+\mathrm{e}$

Transfert de charge
8) $\mathrm{O}^{+}+\mathrm{O}_{2} \rightarrow \mathrm{O}_{2}^{+}+\mathrm{O}$
9) $\mathrm{O}_{2}^{+}+\mathrm{O} \rightarrow \mathrm{O}^{+}+\mathrm{O}_{2}$
10) $\mathrm{O}_{2}^{+}+\mathrm{O}_{2} \rightarrow \mathrm{O}_{3}^{+}+\mathrm{O}$
11) $\mathrm{O}_{2}^{+}+2 \mathrm{O}_{2} \rightarrow \mathrm{O}_{4}^{+}+\mathrm{O}_{2}$

12) $\mathrm{O}^{-}+\mathrm{O}_{2} \rightarrow \mathrm{O}_{2}^{-}+\mathrm{O}$

13) $\mathrm{O}^{-}+\mathrm{O}_{3} \rightarrow \mathrm{O}_{3}^{-}+\mathrm{O}$

14) $\mathrm{O}^{-}+2 \mathrm{O}_{2} \rightarrow \mathrm{O}_{3}^{-}+\mathrm{O}_{2}$

15) $\mathrm{O}_{2}^{-}+\mathrm{O} \rightarrow \mathrm{O}^{-}+\mathrm{O}_{2}$

16) $\mathrm{O}_{2}^{-}+\mathrm{O}_{2} \rightarrow \mathrm{O}_{3}^{-}+\mathrm{O}$

17) $\mathrm{O}_{2}^{-}+\mathrm{O}_{3} \rightarrow \mathrm{O}_{3}^{-}+\mathrm{O}_{2}$

18) $\mathrm{O}_{2}^{-}+2 \mathrm{O}_{2} \rightarrow \mathrm{O}_{4}^{-}+\mathrm{O}_{2}$

19) $\mathrm{O}_{3}^{-}+\mathrm{O}_{2} \rightarrow \mathrm{O}_{2}^{-}+\mathrm{O}_{3}$

20) $\mathrm{O}_{4}^{-}+\mathrm{O} \rightarrow \mathrm{O}_{3}^{-}+\mathrm{O}_{2}$

21) $\mathrm{O}_{4}^{-}+\mathrm{O}_{2} \rightarrow \mathrm{O}_{2}^{-}+2 \mathrm{O}_{2}$

Détachement électrique
22) $\mathrm{O}^{-}+\mathrm{O} \rightarrow \mathrm{O}_{2}+\mathrm{e}$
23) $\mathrm{O}^{-}+\mathrm{O}_{2} \rightarrow \mathrm{O}+\mathrm{O}_{2}+\mathrm{e}$
24) $\mathrm{O}^{-}+\mathrm{O}_{2}\left({ }^{1} \Delta_{\mathrm{g}}\right) \rightarrow \mathrm{O}_{3}+\mathrm{e}$
25) $\mathrm{O}_{2}^{-}+\mathrm{O} \rightarrow \mathrm{O}_{3}+\mathrm{e}$
26) $\mathrm{O}_{2}^{-}+\mathrm{O}_{2} \rightarrow 2 \mathrm{O}_{2}+\mathrm{e}$
27) $\mathrm{O}_{2}^{-}+\mathrm{O}_{2}\left({ }^{1} \Delta_{\mathrm{g}}\right) \rightarrow 2 \mathrm{O}_{2}+\mathrm{e}$

Recombinaison électron-ion

28) e $+\left\{\begin{array}{c}\mathrm{O}^{+} \\ \mathrm{O}_{2}^{+} \\ \mathrm{O}_{3}^{+} \\ \mathrm{O}_{4}^{+}\end{array}\right\} \rightarrow\left\{\begin{array}{c}\mathrm{O} \\ 2 \mathrm{O} \\ \mathrm{O}+\mathrm{O}_{2} \\ 2 \mathrm{O}_{2}\end{array}\right\}$

Recombinaison ion-ion

$$
\text { 29) }\left\{\begin{array}{l}
\mathrm{O}^{-} \\
\mathrm{O}_{2}^{-} \\
\mathrm{O}_{3}^{-} \\
\mathrm{O}_{4}^{-}
\end{array}\right\}+\left\{\begin{array}{l}
\mathrm{O}^{+} \\
\mathrm{O}_{2}^{+} \\
\mathrm{O}_{3}^{+} \\
\mathrm{O}_{4}^{+}
\end{array}\right\} \rightarrow\left\{\begin{array}{c}
\mathrm{O} \\
\mathrm{O}_{2}
\end{array}\right\}
$$

Recombinaison atomique
30) $2 \mathrm{O}+\mathrm{O}_{2} \rightarrow 2 \mathrm{O}_{2}$
31) $3 \mathrm{O} \rightarrow \mathrm{O}+\mathrm{O}_{2}$
32) $\mathrm{O}+2 \mathrm{O}_{2} \rightarrow \mathrm{O}_{2}+\mathrm{O}_{2}$
33) $\mathrm{O}+\mathrm{O}_{3} \rightarrow 2 \mathrm{O}_{2}$
34) $\mathrm{O} \stackrel{\text { paroi }}{\longrightarrow} 1 / 2 \mathrm{O}_{2}$

$k$

$\sigma_{\max }\left(\mathrm{cm}^{2}\right)$

$2,72 \times 10^{-16}$

$1,54 \times 10^{-18}$

$1,0 \times 10^{-16}$

$1,41 \times 10^{-18}$

$4,85 \times 10^{-19}$

$2,25 \times 10^{-18}$

$3,0 \times 10^{-20}$

$2 \times 10^{-11} \mathrm{~cm}^{3} / \mathrm{s}$

$2,8 \times 10^{-30} \mathrm{~cm}^{6} / \mathrm{s}$

$2,5 \times 10^{-14} \mathrm{~cm}^{3} / \mathrm{s}$

à $\mathrm{E} / \mathrm{p}=20 \mathrm{~V} / \mathrm{cm}$ torr

$3,4 \times 10^{-12} \mathrm{~cm}^{3} / \mathrm{s}$

à $\mathrm{E} / \mathrm{p}=45 \mathrm{~V} / \mathrm{cm}$ torr

$5,3 \times 10^{-10} \mathrm{~cm}^{3} / \mathrm{s}$

$1,0 \pm 0,2 \times 10^{-30} \mathrm{~cm}^{6} / \mathrm{s}$

$5 \times 10^{-10} \mathrm{~cm}^{3} / \mathrm{s}$

$4,0 \times 10^{-10} \mathrm{~cm}^{3} / \mathrm{s}$

$3 \times 10^{-31} \mathrm{~cm}^{6} / \mathrm{s}$

$4 \times 10^{-10} \mathrm{~cm}^{3} / \mathrm{s}$

$6 \times 10^{-15} \mathrm{~cm}^{3} / \mathrm{s}$

$<10^{-18}$

$4 \times 10^{-17}$

$3,0 \times 10^{-10} \mathrm{~cm}^{3} / \mathrm{s}$

$\sim 3 \times 10^{-10} \mathrm{~cm}^{3} / \mathrm{s}$

$5,0 \times 10^{-10} \mathrm{~cm}^{3} / \mathrm{s}$

$\sim 2 \times 10^{-10} \mathrm{~cm}^{3} / \mathrm{s}$

$8 \times 10^{-16}$

$1 \times 10^{-16}$

$7 \times 10^{-16}$

$7 \times 10^{-16}$

$\lesssim 10^{-7} \mathrm{~cm}^{3} / \mathrm{s}$

$\sim 10^{-7} \mathrm{~cm}^{3} / \mathrm{s}$

$2,3 \times 10^{-33} \mathrm{~cm}^{6} / \mathrm{s}$

$1,5 \times 10^{-34} \mathrm{~cm}^{6} / \mathrm{s}$

$1,9 \times 10^{-35} \exp (2100 / \mathrm{RT}) \mathrm{cm}^{6} / \mathrm{s}$

$2,0 \times 10^{-11} \exp (-4790 / \mathrm{RT}) \mathrm{cm}^{3} / \mathrm{s}$

$v=1,6 \times 10^{-4}$ à $1,4 \times 10^{-2}$

$\left(T=20-600^{\circ} \mathrm{C}\right)$ 
ce qui invalide toute description du système à partir de la théorie cinétique,

b) les écarts entre les temps caractéristiques des divers processus physiques et chimiques sont faibles et tous les processus doivent généralement être considérés simultanément [116],

c) la cinétique chimique n'est pas indépendante de la cinétique physique, ce qui invalide encore la théorie cinétique,

d) les processus aboutissant à un même état final peuvent être très différents à partir du même état initial, aspect qui complique très sérieusement les calculs de mécanique quantique,

e) les barrières énergétiques telles qu'elles sont définies dans la théorie d'Arrhenius ne sont plus valides en chimie des plasmas du fait des collisions entre états excités.

Dans ces conditions écrire une équation susceptible d'être la base de la cinétique chimique hors d'équilibre est une tâche complexe. Une telle équation peut être celle de Liouville, de Pauli, de Boltzmann ou tout autre. L'équation de Liouville a l'avantage d'être l'intégrale des équations de la mécanique mais elle est très difficilement applicable du fait de l'irréversibilité des évolutions. Les équations de Pauli et de Boltzmann nécessitent des calculs numériques sur ordinateur mais elles sont les seules susceptibles de conduire à une solution [116] concrète.

L'équation de Pauli s'exprime en fonction des coefficients : de transport (plasma inhomogène), de transition radiative spontanée, d'ionisation par collision, de recombinaison par collision, d'excitation par collision, de désexcitation par collision, de recombinaison radiative spontanée. La résolution est très complexe même en supposant le gaz monoatomique avec une seule espèce excitée comme le montrent par exemple les calculs de Catherinot [117] et de Shaw [118] pour un plasma inhomogène et ceux de Capitelli [119] pour un plasma homogène.

L'étude de ces processus hors d'équilibre pour laquelle il reste beaucoup à faire est fondamentale non seulement pour les phénomènes que l'on peut observer en chimie des plasmas à basse pression c'està-dire dans un milieu hors d'équilibre (cf. par exemple $[120,121])$ mais encore pour les réactions dans des plasmas thermiques où le réacteur de trempe (cf. $\S 6.4)$ joue un rôle capital.

\subsection{LES ÉQUILIBRES CHIMIQUES ET LES FONCTIONS} THERMODYNAMIQUES. - La thermodynamique classique peut être appliquée aux systèmes de la chimie des plasmas, mais son rôle est très ambigu : elle s'avère en effet être un guide précieux dans la prévision des réactions, mais les multiples phénomènes de déséquilibre et de relaxation peuvent la rendre inefficace et entacher les calculs d'imprécisions et même d'erreurs non négligeables.
On utilise généralement le diagramme $\Delta G=f(T)_{\mathrm{p}}$ qui illustre la stabilité des réactions et conduit facilement à la détermination des constantes d'équilibre. La connaissance de ces grandeurs thermodynamiques permet le calcul des concentrations du système supposé en équilibre à toutes les températures. Nombre d'études ont été ainsi menées, par exemple pour les systèmes hydrogène-hydrocarbure [122], carbone-hydrogène, oxygène-azote [123-126], azotezirconium [127-129], azote-silicium, azote-aluminium [130-131]. Il est alors intéressant de comparer ces calculs aux résultats expérimentaux obtenus par trempe des systèmes en équilibre. Les auteurs qui ont tenté de telles comparaisons ont obtenu des accords médiocres. Ils ont donc été amenés à corriger les résultats obtenus, par approximations successives, afin de prendre en considération des phénomènes inapparents dans cet ensemble thermodynamique à l'équilibre (cf. $\S 6.1$ ).

Ainsi nous résumerons en disant que les données de la thermodynamique classique forment une base utile à la prévision des réactions, avec des réserves quant aux résultats quantitatifs si elles indiquent leur succès. Cependant, en dépit de ces obstacles, ces études d'équilibre sont souvent à l'origine d'améliorations importantes au niveau de la technologie.

Lorsque l'on choisit les variables température, pression, le système est caractérisé par la fonction enthalpie libre de Gibbs $G$ qui s'exprime en fonction des potentiels chimiques $\mu$ et des concentrations molaires $n$ des espèces $i$ dans la phase $\alpha$ par :

$$
G=\sum_{i=1}^{n} \sum_{\alpha=1}^{\emptyset} \mu_{i}^{\alpha} n_{i}^{\alpha} .
$$

Pour un système isolé, décrit par les variables $T, P$ et $n_{i}^{\alpha}$, l'évolution vers l'état d'équilibre est caractérisée par l'inégalité $\mathrm{d} G<0$, l'équilibre étant atteint lorsque $\mathrm{d} G=0$. Le problème du calcul d'un équilibre chimique à température et pression constante consiste donc à trouver l'ensemble des $n_{i}^{\alpha}(i=1, \ldots, N$; $\alpha=1, \ldots, \varnothing)$ tels que $\mathrm{d} G=0$ compte tenu de la loi de conservation des éléments.

De nombreux auteurs, étrangers pour la plupart [132], ayant publié des études générales sur ce sujet, s'accordent sur le fait que les techniques de résolution peuvent être classées en deux grands groupes : les méthodes d'optimisation, consistant en la recherche de l'extrémum d'une fonction caractéristique, constituent la première catégorie, tandis que celles $\mathrm{du}$ second groupe se ramènent toutes, après différents calculs, à la résolution d'un système non linéaire. Il existe par ailleurs quelques autres méthodes que l'on ne peut classer dans l'un ou l'autre de ces groupes. On trouvera par exemple chez Bourdin [131] une comparaison détaillée de deux méthodes : la méthode de Brinckley [133 à 135] et la méthode de la NASA [136]. Enfin la résolution des différents systèmes d'équations linéarisées implique que l'on 
connaisse les potentiels chimiques $\mu$ qui s'expriment [137] en fonction des fonctions de partition dont le calcul à partir des données spectroscopiques est loin d'être simple [138].

A titre d'exemple nous avons représenté sur les figures 6 et 7 l'évolution, en fonction de la tempéra-

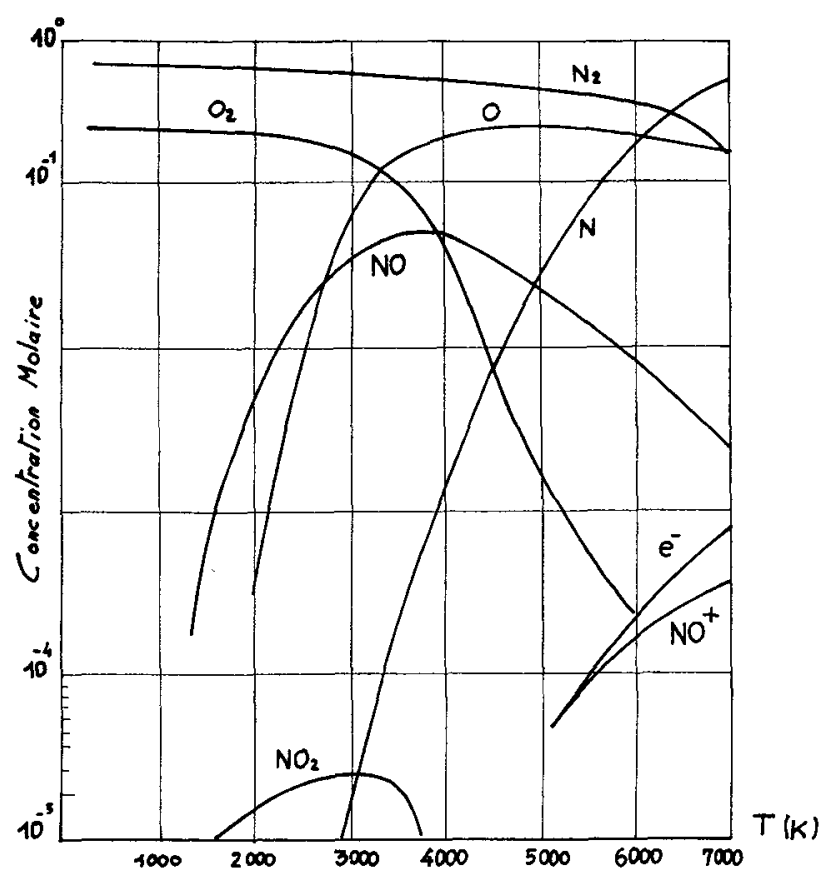

FIG. 6. - Composition de l'air à l'équilibre (Ammann, Timmins Aiche J. 12 1966).

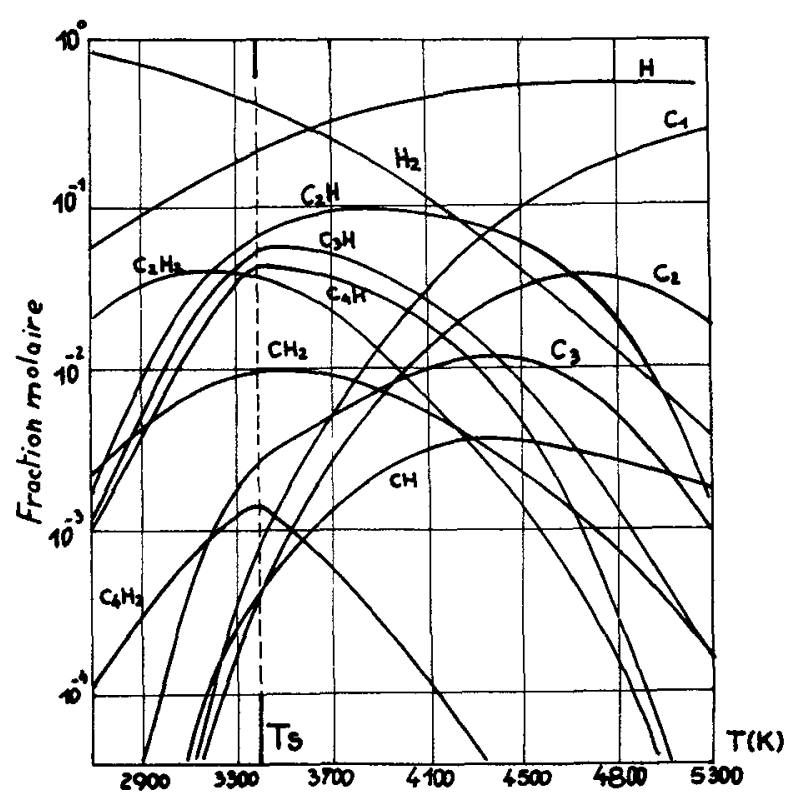

FıG. 7. - Diagramme d'équilibre $\left(\mathrm{C} / \mathrm{H}_{2}=1,0\right)$ (Baddour-Blanchet).

ture, de la composition à la pression atmosphérique d'un plasma d'air [96] et un plasma carbone-hydrogène [139]. Si l'on cherche à obtenir $\mathrm{NO}$ ou $\mathrm{C}_{2} \mathrm{H}_{2}$ nous voyons sur ces figures que les températures idéales sont respectivement $3500 \mathrm{~K}$ environ et $3300 \mathrm{~K}$.
A ces températures les concentrations molaires maximales de $\mathrm{NO}$ et $\mathrm{C}_{2} \mathrm{H}_{2}$ sont respectivement de $5,2 \%$ et de $21 \%$. Or dans la pratique, compte tenu des réacteurs de trempe, on obtient dans des conditions similaires, des pourcentages qui peuvent aller jusqu'à $14 \%$ pour NO et $46 \%$ pour $\mathrm{C}_{2} \mathrm{H}_{2}$, c'est dire si la recombinaison lors de la trempe des espèces atomiques telles que $\mathrm{N}$ et $\mathrm{O}$ ou des divers radicaux carbonehydrogène participe de façon prépondérante à la réaction globale au cours des processus cinétiques, aussi rapides soient-ils.

6.3 LES PROPRIÉTÉS DE TRANSPORT. - Dans les réactions hétérogènes en plasma, par exemple nitruration directe de l'aluminium ou du silicium [140] ou en métallurgie extractive [141], les réactions sont d'abord fonction des échanges thermiques entre plasma et particules solides et donc, entre autres, de la conductivité thermique et des coefficients de diffusion des plasmas utilisés.

Les phénomènes que l'on est amené à prendre en considération sont la diffusion, la viscosité, la conductivité thermique, la conductivité électrique, lesquels du point de vue de la Physique, sont tous semblables en ce sens qu'ils font intervenir le transport d'une certaine propriété au sein d'un gaz [142]. L'étude théorique en cinétique physique classique des coefficients de transport d'un plasma se fait à l'aide de l'équation intégro-différentielle de Boltzmann [143, 144]. Cette théorie conçue pour des gaz monoatomiques, à des températures relativement basses, doit être aménagée [142 à 144] pour tenir compte des hautes températures et de la présence de molécules, d'atomes excités [145] et d'ions [146] à l'intérieur du plasma. Ainsi que l'a mis en évidence Athye [146] et que l'a démontré Devoto [147 à 151] pour un plasma d'hydrogène, d'argon, d'hélium, de krypton et de xénon, les résultats pour être valables doivent être basés sur une résolution à un ordre plus élevé de l'équation de Boltzmann, par la méthode de Chapman-Enskog, à l'intérieur de la première approximation d'Enskog. D'après les conclusions de Devoto l'ordre quatre paraît nécessaire dans certains cas.

Pour le calcul des coefficients de transport les divers auteurs s'inspirent généralement des travaux de Devoto [147]. Cependant l'existence d'échanges d'énergie entre les degrés internes de liberté [152] et le fait que le plasma considéré soit le siège d'un certain nombre de réactions chimiques [153] entraînent l'adjonction de deux termes correctifs à la conductibilité thermique (de translation) prolongée par Devoto. Ces derniers ne peuvent malheureusement être calculés qu'à l'ordre un.

A l'aide de la fonction de distribution des vitesses le calcul des propriétés de transport peut alors être développé en utilisant l'équation de Boltzmann [154, $155]$ mais il reste toutefois deux importants problèmes à régler :

- l'évaluation des intégrales de collision qui appa- 
raissent lors de la résolution de l'équation de Boltzmann par la méthode de Chapman-Enskog [142], - pour de faibles degrés d'ionisation la convergence de la solution de Chapman-Enskog est mauvaise, mais lorsque l'on veut augmenter l'ordre de l'approximation on augmente en même temps le nombre d'intégrales de collision à calculer [155].

La mauvaise convergence de la solution de Chapman-Enskog est généralement imputée à l'existence de forces d'interaction à longue distance. Sandler et Mason [156] ont montré que ces difficultés de convergence apparaissent également pour les mélanges de particules neutres (caractérisés par des interactions à courte distance) lorsqu'ils sont constitués de particules de masses très différentes.

Les résultats obtenus à partir de ces propriétés de transport sont particulièrement intéressants en chimie des plasmas dès qu'il s'agit de considérer des mélanges de gaz. En effet, par exemple, l'adjonction d'hydrogène à un plasma d'argon modifie beaucoup la conductivité thermique [157] et par conséquent les possibilités d'échange thermique entre le plasma et les corps que l'on veut y faire réagir. Le pic qui apparait à la température de dissociation de l'hydrogène (Fig. 8) peut ainsi multiplier la conductivité thermique du plasma d'argon dans les rapports de 1 à 100 suivant le pourcentage d'hydrogène utilisé, cette modification a d'autant plus d'intérêt qu'elle intervient vers $3500^{\circ}$, température souvent rencontrée dans les plasmas thermiques, et surtout dans les systèmes chimiques azote-oxygène, carbóne-hydrogène... (cf. § 5.2).

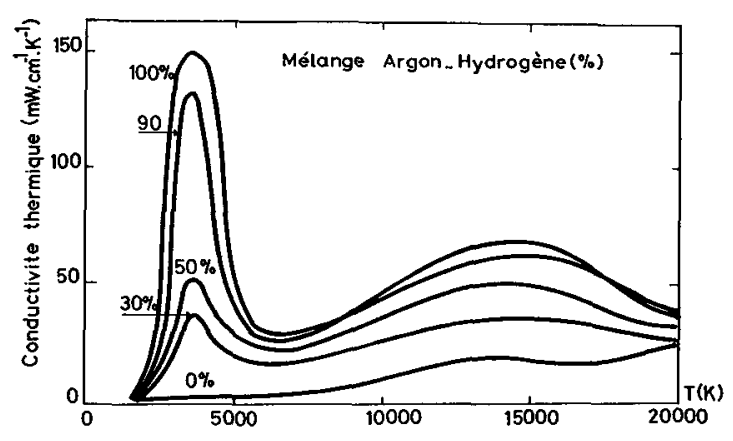

FIG. 8. - Conductivité thermique $\left(\mathrm{mW} \cdot \mathrm{cm}^{-1} \cdot \mathrm{K}^{-1}\right)$.

6.4 LA TREMPE. - Dans pratiquement tous les cas, il est indispensable d'effectuer cette trempe afin de figer les produits avant que des réactions inverses ne les détruisent. (Nous parlons ici de système figé, mais de nombreux dispositifs de trempe ne réalisent pas exactement cette fonction, ils se situent en général à mi-chemin entre le cas figé et le cas d'équilibre.)

De nombreuses techniques ont été proposées avec des vitesses de trempe $v$ comprises entre $10^{5}$ et $10^{8} \mathrm{~K} / \mathrm{s}$ :

- refroidissement homogène par expansion adiabatique dans une tuyère de Laval [158] $\left(v=10^{7} \mathrm{~K} / \mathrm{s}\right)$,
- contact avec des solides refroidis $\left(v=10^{8} \mathrm{~K} / \mathrm{s}\right.$ pour un mélange hydrogène-hydrocarbure $[158,159]$; $v \simeq 3,5 \times 10^{7} \mathrm{~K} / \mathrm{s}$ pour le système oxygène-azote [96], [160,161]),

- combinaison des effets précédents en utilisant des tuyères de Laval refroidies,

- refroidissement par pulvérisation de liquide, par exemple l'eau ou les hydrocarbures pour la synthèse de l'acétylène (voir aussi $[160,162])$. Ce procédé présente l'avantage ou l'inconvénient suivant les cas d'introduire une nouvelle espèce chimique susceptible de réagir avec les produits déjà formés,

- prise en charge des produits par un gaz froid, Grey et Jacobs par exemple ont évalué les vitesses de trempe par l'hélium à la température ambiante sur des produits synthétisés dans un plasma d'argon [163],

- enfin n'oublions pas que la réaction chimique elle-même peut être un facteur de trempe important lors des réactions endothermiques.

Aucune analyse comparative de ces différentes méthodes n'a été effectuée, il est donc difficile de préciser leur efficacité relative, autrement que par la comparaison de leur vitesse (en $\mathrm{K} / \mathrm{s}$ ).

A titre d'exemple reprenons rapidement les résultats obtenus par Timmins et Amman [96] à propos $\mathrm{du}$ système azote-oxygène pour lequel, comme nous l'avons vu pour les équilibres chimiques, la concentration d'équilibre maximale de NO est atteinte à $3500 \mathrm{~K}$ $(5,2 \%)$ sous une atmosphère. Leurs calculs, compte tenu d'un certain nombre de réactions et de la valeur estimée des coefficients de celles-ci, montrent (Fig. 9)

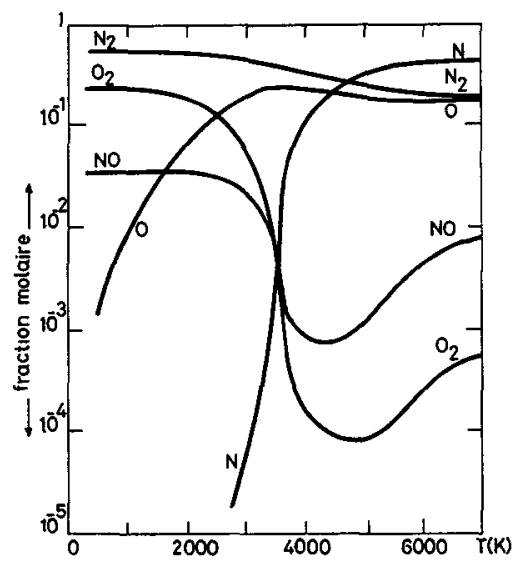

FIG. 9. - Trempe rapide de l'air à $7000 \mathrm{~K}$.

effectivement que les espèces atomiques telles que $\mathrm{N}$ et $\mathrm{O}$ participent grandement à la création de NO au cours de la trempe. L'influence des phénomènes de recombinaison à la paroi est importante au-delà de $4000 \mathrm{~K}$. Il faut aussi noter que la sélection et la mise en cuvre des processus de trempe sont déterminantes pour le rendement des réactions. Par exemple Polak [116] indique que pour la conversion du méthane en acétylène un retard de la trempe de $2 \times 10^{-3} \mathrm{~s}$ entraîne une diminution de l'acétylène de $15,5 \%$ 
à $10 \%$ et de même pour les oxydes de l'azote une diminution de la vitesse de trempe de $10^{8}$ à $10^{7} \mathrm{deg} / \mathrm{s}$ conduit à une diminution de $\mathrm{NO}$ de 9,6 à $6,4 \%$. En fait d'une manière générale il est nécessaire de suivre une loi de trempe de la forme $\mathrm{d} T / \mathrm{d} t=f(T)$ bien définie. Même si de tels calculs sont encore loin de la réalité expérimentale ils permettent cependant un début d'interprétation des résultats obtenus.

6.5 Les TRANSFerTS DE CHALEUR ET DE Matière Plasma-ParTiCules et Plasma-Parois. - Le système des équations de transfert de chaleur entre un écoulement de plasma et une phase condensée est complexe du fait de l'interaction des phénomènes de conduction, convection et radiation. On est conduit à des équations intégro-différentielles non linéaires à coefficients variables (les propriétés physiques du plasma ne sont en effet pas homogènes).

De plus on peut avoir transfert de masse en présence ou non de réactions chimiques, ce qui conduit à l'apparition de termes puits de chaleur, de la diffusion enthalpique (différence d'enthalpie entre les espèces diffusantes) et du couplage chaleur-matière (thermodiffusion). Il n'est donc pas possible d'aborder le problème dans sa totalité. On peut par contre, analyser la littérature à ce sujet, choisir un modele réaliste et simple et éventuellement compléter par des mesures les informations disponibles.

Dans cet esprit on est alors amené à utiliser les équations de l'analyse dimensionnelle [164-168] en utilisant les nombres adimensionnels usuels et les équations de transport adimensionnelles de chaleur et de masse [169-170].

6.5.1 Transfert de chaleur à la paroi (fixe ou tournante). - Il convient de prendre en compte les variations importantes avec les gradients de température des propriétés thermodynamiques et de transport [169] ainsi que les phénomènes hors d'équilibre au voisinage des parois. De plus on suppose généralement l'écoulement laminaire [170] et on adopte pour la résolution des équations des approximations semblables à celles que l'on admet dans la couche limite ou dans les traînées en mécanique des fluides [170 à 173] en négligeant les forces axiales vis-à-vis des forces radiales. Il faut également prendre en compte les apports d'énergie au voisinage de la cathode [174, 175] et de l'anode [176-180], la pression magnétique $[181,182]$, le rayonnement, la convection $[183,184] \ldots$ En fait les hypothèses principales faites pour écrire ces équations de transfert plasma sont les suivantes [185-187] :

1) Ecoulement laminaire.

2) Ecoulement stable et stationnaire.

3) Ecoulement symétrique par rapport à l'axe.

4) Inexistence de tourbillons et vitesse périphérique nulle.

5) Plasma en état d'équilibre thermodynamique local (ETL).
6) Loi d'Ohm applicable.

7) Dissipation de viscosité négligeable (diffusion de masse).

8) Champ magnétique self-induit négligeable.

9) Courant dans la direction radiale négligeable.

10) Conduction de chaleur axiale négligeable.

11) Gradient de pression radial négligeable.

12) Gradient de potentiel radial négligeable.

13) Champ électrique uniforme dans chaque plan perpendiculaire à la direction axiale.

14) Plasma optiquement mince.

15) Facteur d'émission de la paroi égal à l'unité (corps noir).

D'une manière générale tous ces calculs théoriques sous-estiment le rôle du rayonnement. Signalons par exemple à ce propos les résultats expérimentaux obtenus à Odeillo [188] pour un plasma d'azote de nombre de Reynolds R compris entre 300 et 3000 (l'hypothèse de l'écoulement laminaire devient douteuse) qui conduisent à une relation linéaire

$$
N u_{x}=a \operatorname{Re}_{x}
$$

où $N u_{x}$ est le paramètre d'échange de chaleur local global (conduction, convection, rayonnement) et $a$ une constante de l'ordre de $10^{-2}$ qui dépend de la vitesse de rotation de la paroi.

6.5.2 Transferts de chaleur plasma-particules. La suspension au sein du plasma d'un matériau très divisé perturbe complètement ses propriétés [189192], de plus il est fondamental de traiter simultanément les transferts convectifs et radiatifs [193] et de tenir compte du couplage entre les phénomènes de transport [194]. On écrit alors [195] les équations de conservation dans le cas d'un système pseudo-binaire dont les deux constituants ne réagissent pas entre eux et l'on suppose souvent la convection forcée négligeable et le milieu optiquement mince. De plus on suppose que :

- les particules sont sphériques et rigides,

- les variations de rayon n'interviennent qu'au second ordre,

- la pression et l'enthalpie de rayonnement sont négligeables,

- le travail des forces extérieures massiques est négligeable,

- les nombres d'Eckert et de Reynolds sont petits,

- le plasma est infini et isotrope.

Les divers auteurs [195] aboutissent alors à une corrélation semi-empirique de la forme :

$$
N u=2\left[1+a \operatorname{Re}^{m} \operatorname{Pr}^{n}+b \mathrm{Gr}^{m^{\prime}} \operatorname{Pr}^{n^{\prime}}\right]
$$

où $\mathrm{Pr}$ et $\mathrm{Gr}$ sont respectivement les nombres de Prandt et de Grashof [167].

Cependant il n'est pas spécifié à quelles températures doivent être calculés les paramètres adimensionnels et $m, n, m^{\prime}, n^{\prime}$ dépendent des valeurs de $\operatorname{Re}$ et du régime hydrodynamique. 
Les différents choix que l'on peut alors faire sont les suivants :

- température de la particule,

- température moyenne du film (de la couche limite thermique),

- température du plasma à l'infini, à l'extérieur de la couche limite thermique.

Ces choix affectent profondément la valeur des paramètres adimensionnels.

Aussi imparfaits et délicats que puissent être ces calculs, ils donnent cependant des renseignements précieux sur l'évolution de la température des particules dans un plasma en fonction de leurs diamètres et de la température du plasma comme le montre par exemple la figure 10 extraite de [195] relative à des particules de zircone. On conçoit alors facilement que pour le traitement des particules dans un plasma le prọblème de la granulométrie disponible soit fondamental pour le rendement du réacteur.

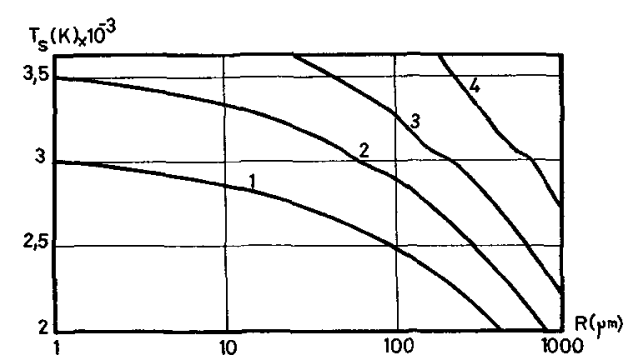

Fig. 10. - Evolution de la température d'une particule de $\mathrm{ZrO}_{2}$ en fonction de son diamètre pour diverses températures du plasma : $1: 3000 \mathrm{~K} 2: 7500 \mathrm{~K}, 3: 8000 \mathrm{~K}, 4: 9000 \mathrm{~K}$.

7. Généralités sur la réalisation du réacteur en chimie des plasmas. - Un réacteur de chimie en plasma est un tout qui doit être adapté à la réaction désirée mais que l'on divise en trois parties pour des questions de commodité de raisonnement : génération du plasma, réactions chimiques, trempe.

La figure 11 donne un schéma théorique de ces trois séquences, leur ordre possible ainsi que leur rôle [61]. Un des principaux problèmes est celui du mélange des réactifs avec le plasma qui conformément au schéma peut se faire avant ou après le générateur.

a) Mélange avant le générateur. - L'adoption de cette technique permet d'éviter les problèmes que posent les mélanges de produits froids avec le plasma. Cependant, ce prémélange n'est pas utilisable lorsque l'un des produits injectés est susceptible de réagir avec les électrodes ou les parois, il pose également quelques problèmes technologiques dans le cas des réactifs solides. Par ailleurs, il n'est pas toujours nécessaire d'exposer aussi violemment les réactifs au plasma.

b) Mélange après le générateur. - On peut aussi mélanger le produit $B$ avec le plasma $A$ (ou contenant A) après le générateur, les recombinaisons (A-B) s'effectuent alors dans le jet ou son panache. Le flux

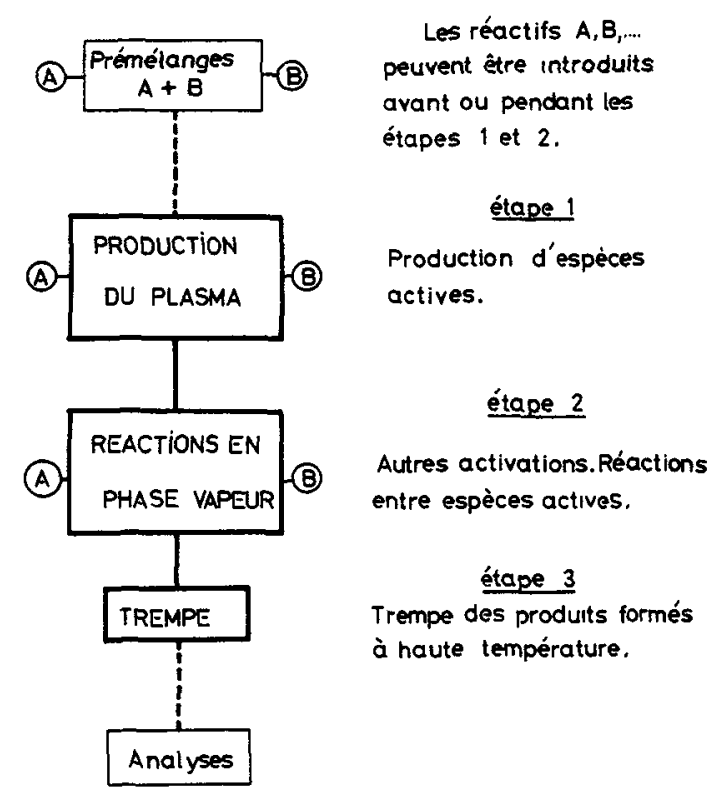

FIG. 11. - Schéma synoptique d'une réaction en plasma-chimie.

réactif $\mathbf{B}$, à basse température, perturbe alors le plasma, perturbation qui est fonction des propriétés physiques de $B$, on est alors généralement en présence d'un système réactif en déséquilibre de température.

L'introduction des produits dans ou après le générateur pose souvent des problèmes technologiques, aussi de nombreuses études ont été faites, afin de déterminer le mode optimal d'injection [130, 196, 197]. Des systèmes originaux ont été proposés, tels que des cathodes creuses ou même poreuses [198] et les anodes volatiles [199, 200], l'injection de liquide est généralement réalisée avec un atomiseur ultrasonique [201].

Dans le cas des plasmas froids, la réaction a lieu au sein du plasma au niveau des champs électromagnétiques. L'excitation directe des atomes ou molécules par collision électronique est alors, dans la majeure partie des cas, le mécanisme principal de création des états excités. Les réactions ont un caractère sélectif dans la mesure où seules quelques espèces participent effectivement à la formation du composé recherché. Il s'agit alors pour obtenir un bon rendement chimique de favoriser l'excitation des espèces utiles. On peut se rendre maître de cette chimie sélective (comparable en de nombreux points à la photochimie) en jouant sur la puissance de génération, mais aussi en cherchant à utiliser les niveaux métastables des gaz [126]. Par ailleurs, sachant quels sont les niveaux (et les espèces) à exciter, il est indispensable de connâ̂tre la cinétique des différentes réactions élémentaires de façon à pouvoir établir un bilan d'excitation et de formation.

Ces processus sélectifs qui ont un caractère absolument fondamental au niveau des plasmas froids, jouent également un rôle important dans les plasmas 
thermiques. Dans ces derniers, les modes d'excitation sont souvent plus divers et ont généralement des cinétiques très différentes puisque situés à des températures et pressions beaucoup plus élevées.

Dans les plasmas thermiques, en supposant que le mélange des réactifs soit suffisamment rapide, on peut en première approximation considérer le mélange comme un gaz en équilibre à haute température. On peut alors, à l'aide des lois classiques, calculer la concentration des différentes espèces en ne tenant compte dans un premier temps que des seuls effets thermiques. Le bilan des réactions à haute tempéra-

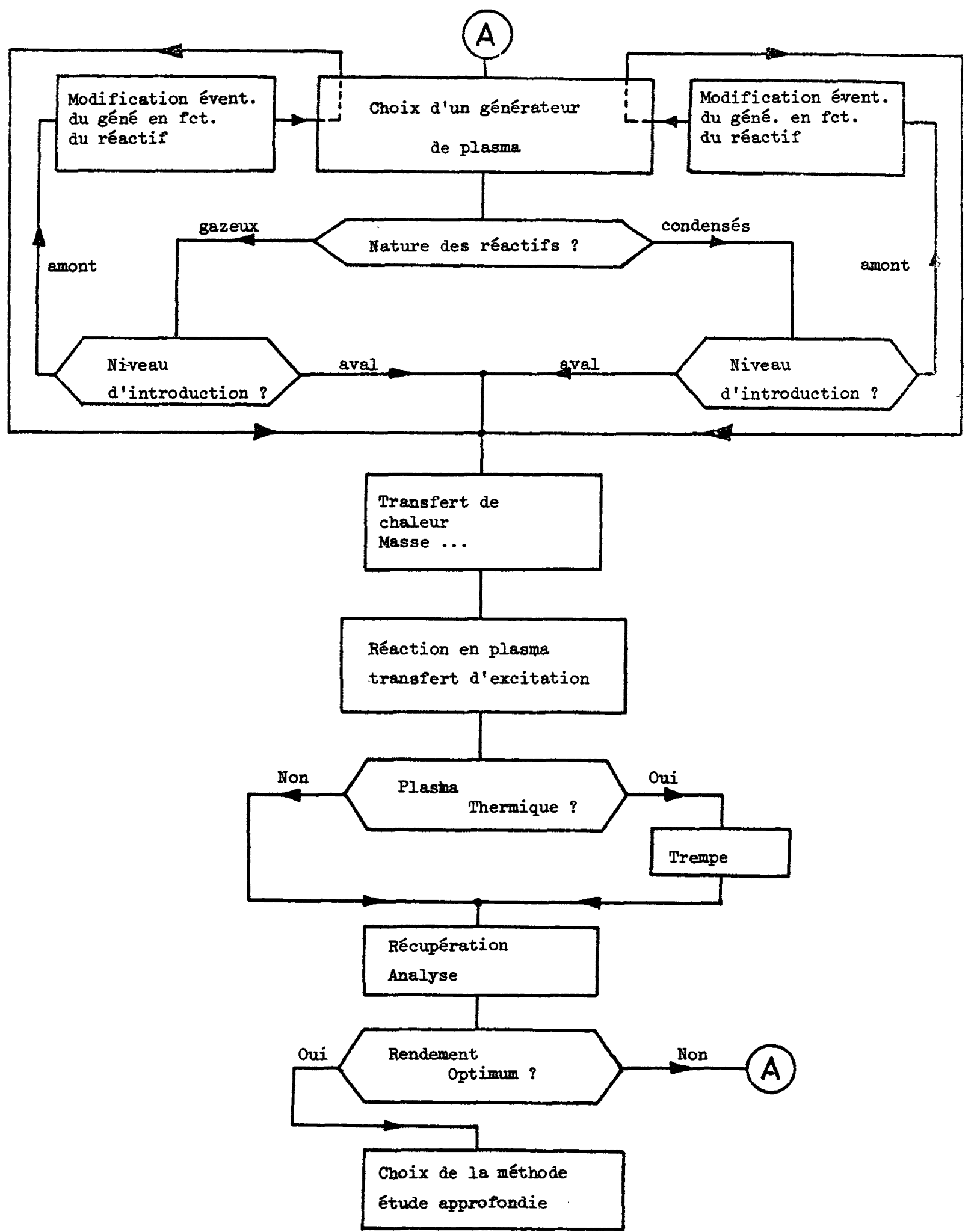

Fig. 12. - Organigramme représentant les diverses étapes du choix d'un réacteur en chimie du plasma. 
ture est alors simple. Mais les espèces ainsi formées doivent être conservées à une température donnée et figées selon une loi $T(t)$ bien définie. Quels seront donc les effets de cette trempe ? Sert-elle simplement à figer le mélange haute température, ou a-t-elle un effet secondaire sur la composition du mélange? Les réponses que l'on peut apporter à ces problèmes sont encore plus loin d'être satisfaisantes $(\S 6.4)$. Tout d'abord, cela dépend essentiellement du mode de trempe adopté, et par ailleurs l'influence de la fonction $T(t)$ est prépondérante. On trouve en réalité très peu de résultats dans ce domaine qu'il est indispensable de développer car le contrôle complet des réactions en plasma thermique en dépend en grande partie.

L'idéal en plasma chimie serait de pouvoir déterminer le comportement d'un quelconque système chimique en fonction des répartitions énergétiques, de la (ou des) température(s) et de la pression. On pourrait alors concevoir le réacteur le mieux adapté à la synthèse recherchée et enfin l'optimiser en fonction de ses performances réelles. Ce cheminement très satisfaisant est malheureusement encore tout à fait utopique étant donné la complexité et l'interconnexion des processus à traiter.

La première des hypothèses simplificatrices utilisée consiste en général à se fixer sur un seul type de plasma à caractéristiques bien définies, de façon à ce que certains de ces processus se voient naturellement écartés. Mais ce genre d'attitude exclut la possibilité de choix d'un réacteur optimal (ce choix sera donc fait $a$ posteriori après plusieurs essais). On peut résumer sur la figure 12 la logique du cheminement à suivre au cours de l'étude d'une synthèse chimique en plasma.

\section{Remarques sur l'utilisation industrielle des plas-} mas. - Après une période d'euphorie qui a débuté vers les années 1960 durant laquelle on pensait que la recherche en chimie des plasmas allait faire faire un grand bond à l'industrie chimique, l'intérêt des industriels pour celle-ci semble s'être beaucoup tempéré au cours de ces dernières années. Cela est du, semble-til, aux recherches tous azimuts menées en chimie des plasmas sans que des buts logiques aient été définis et des estimations d'échelles et de coût économiques aient été faites. Or dans une économie libérale, il semble bien qu'on ne puisse trouver des crédits pour développer une science nouvelle que dans la mesure où l'on peut mettre en évidence un profit potentiel à partir des recherches développées. Il est donc important, pour le chercheur, de garder présentes à l'esprit un certain nombre d'idées directrices qui sont celles qui seront inévitablement discutées par les industriels au moment où les efforts de recherche auront permis d'atteindre un niveau tel qu'il faille, pour les poursuivre, augmenter l'échelle des réacteurs et donc les fonds. Si nous reprenons l'étude de Wilks [202] il semble que, sur le plan économique, les éléments suivants doivent être considérés pour évaluer la potentialité industrielle d'une réaction donnée en chimie des plasmas, ce sont : la simplicité de production, la qualité du produit, la constance de la qualité et la demande pour le produit fini.

La simplicité est très importante car du simple point de vue des probabilités, plus grand est le nombre d'opérations qui doivent être effectuées correctement, plus grande est la probabilité pour que quelque chose aille mal; donc à toutes les phases d'un processus chimique industriel, on peut dire qu'un processus simple est un bon processus et qu'un bon processus est un processus simple.

Pour le rendement, il faut se souvenir que compte tenu des énergies mises en jeu et du prix actuel de l'énergie électrique, les opérations en plasma ne sont pas particulièrement bon marché. Or à un niveau quelconque du processus $100 \%$ de la matière première passe dans le plasma, donc si cette étape donne $100 \%$ de produit fini ou presque le processus est excellent, si on ne récupère que $50 \%$ du produit fini, le coût de l'opération est en première approximation immédiatement doublé. En fait, ce qui est très important c'est le rapport du produit obtenu au produit de base injecté, mais il est très difficile, $a$ priori, d'affirmer sans une étude économique poussée qu'avec un rapport de $10 \%$ par exemple un processus plasma ne puisse pas encore être économiquement viable.

Dans le domaine de la qualité du produit, le plasma est d'autant plus compétitif que dans certains cas il est seul susceptible de produire des matériaux n'ayant pas d'équivalence. Dans ces conditions un processus plus complexe et des coûts de production plus élevés peuvent être facilement tolérables.

Il est également très important de s'assurer que le produit de base est disponible dans une qualité constante et uniforme et s'il est possible de réaliser un réacteur permettant, à chaque fraction du produit injecté, de récupérer à chaque instant la même température et la même enthalpie. Si les réponses à ces questions sont positives la constance de la qualité est alors remarquable et les opérations plasmas ont un avantage considérable sur les systèmes à haute température conventionnels.

On doit aussi se rappeler que si la nécessité de traitement à haute température existe par exemple pour un matériau du commerce ou s'il s'agit de se procurer un matériau ultra-pur, il existe une opportunité pour qu'un processus basé sur le plasma réponde à la demande.

Enfin pour tous les traitements à haute température le fait qu'un réacteur plasma puisse être mis en route très rapidement et arrêté tout aussi rapidement le rend irremplaçable pour tous les traitements de matériaux dont la demande présente des à-coups $\mathrm{du}$ fait de raisons économiques, saisonnières...

Il reste alors, si les réponses aux questions précédentes sont positives, à faire une analyse des coûts de fabrication $(\mathrm{kWh} / \mathrm{kg})$ et des coûts annexes comme le souligne par exemple Amouroux [203]. 
Réacteur :

\begin{tabular}{|c|c|c|}
\hline Bilan financier & Bilan chimique & Bilan thermique \\
\hline Investissement & Choix de la source & $\begin{array}{l}\text { Rendement énergé- } \\
\text { tique }\end{array}$ \\
\hline $\begin{array}{l}\text { Coût des matières pre- } \\
\text { mières }\end{array}$ & $\begin{array}{l}\text { Choix des réactifs et } \\
\text { du fluide plasma- } \\
\text { gène }\end{array}$ & \\
\hline $\begin{array}{l}\text { Coût d'investissement } \\
+ \text { entretien }\end{array}$ & $\begin{array}{l}\text { Réacteur (configura- } \\
\text { tion, alimentation, } \\
\text { taux de conversion) }\end{array}$ & $\begin{array}{l}\text { Bilan énergétique } \\
\text { partiel }\end{array}$ \\
\hline $\begin{array}{l}\text { Coût des fluides de } \\
\text { trempe }\end{array}$ & $\begin{array}{l}\text { Réacteur de trempe } \\
\text { (alimentation, taux } \\
\text { de conversion) }\end{array}$ & Bilan énergétique \\
\hline Coût $\mathrm{g}$ & Rendementchimique & $\begin{array}{l}\text { Rendement énergé- } \\
\text { tique }\end{array}$ \\
\hline
\end{tabular}

Dispositifs annexes. - Unités de prétraitement des réactifs, d'affinage des produits, aires de stockage, unités de contrôle, unités de traitement des eaux et des fumées...

Le coût de ces dispositifs annexes étant naturellement corrélé aux coûts du réacteur. Par exemple le broyage du minerai pour obtenir une gamme de granulométrie très serrée peut entraîner un coût quatre fois supérieur à celui du même broyage pour une gamme de granulométrie plus large alors que le taux de conversion dans le réacteur varie de moins de $5 \%$.

9. Exemples d'applications industrielles. - Nous nous limiterons à quelques exemples d'application ayant débouché à l'échelle pilote ou mếme industrielle c'est-à-dire à des systèmes dont la production peut se chiffrer en $\mathrm{kg} / \mathrm{h}$ et en $\mathrm{m}^{3} / \mathrm{h}$, les puissances dissipées variant de 0,5 à $10 \mathrm{MW}$.

Les réacteurs plasma utilisés sont généralement soit du type à arc ouvert où l'arc éclate entre deux conducteurs, soit du type générateurs à arc soufflé en courant continu avec superposition de puissance soit en continu soit en alternatif [204, 205]. On utilise guère les plasmas à induction [206] que pour la préparation de matériaux ultra-purs du fait de leur faible niveau de puissance et du rendement énergétique médiocre pour la production du plasma. Aussi avant de décrire quelques procédés industriels : obtention de la zircone à partir du zircon, métallurgie extractive du fer et des ferroalliages, fours de refusion, synthèse de l'acétylène, nous allons rappeler brièvement le principe des arcs utilisés.

9.1 ARCS ET GÉNÉRATEURS A FORTE PUISSANCE. Un des points essentiels qu'il convient d'étudier est celui du contact entre le plasma et le matériau à traiter qu'il soit sous forme gazeuse (mélange), liquide ou solide (temps de séjour). Les principaux types de solutions sont les suivants :

- injection au voisinage de la cathode (effet Maecker) [207] (Fig. 13).

- épanouissement de l'arc par rotation soit à l'aide d'un champ magnétique, soit par effet aérodynamique [208], soit par rotation des électrodes [209] (Fig. 14), deux ou trois effets pouvant naturellement

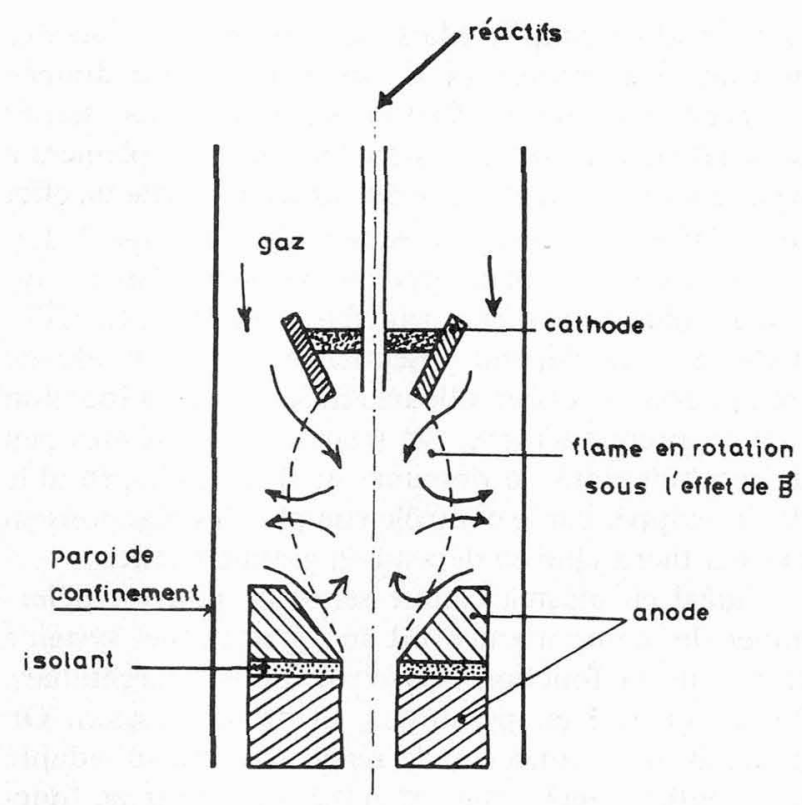

Fig. 13. - Structure possible d'un four utilisant l'effet Maecker.

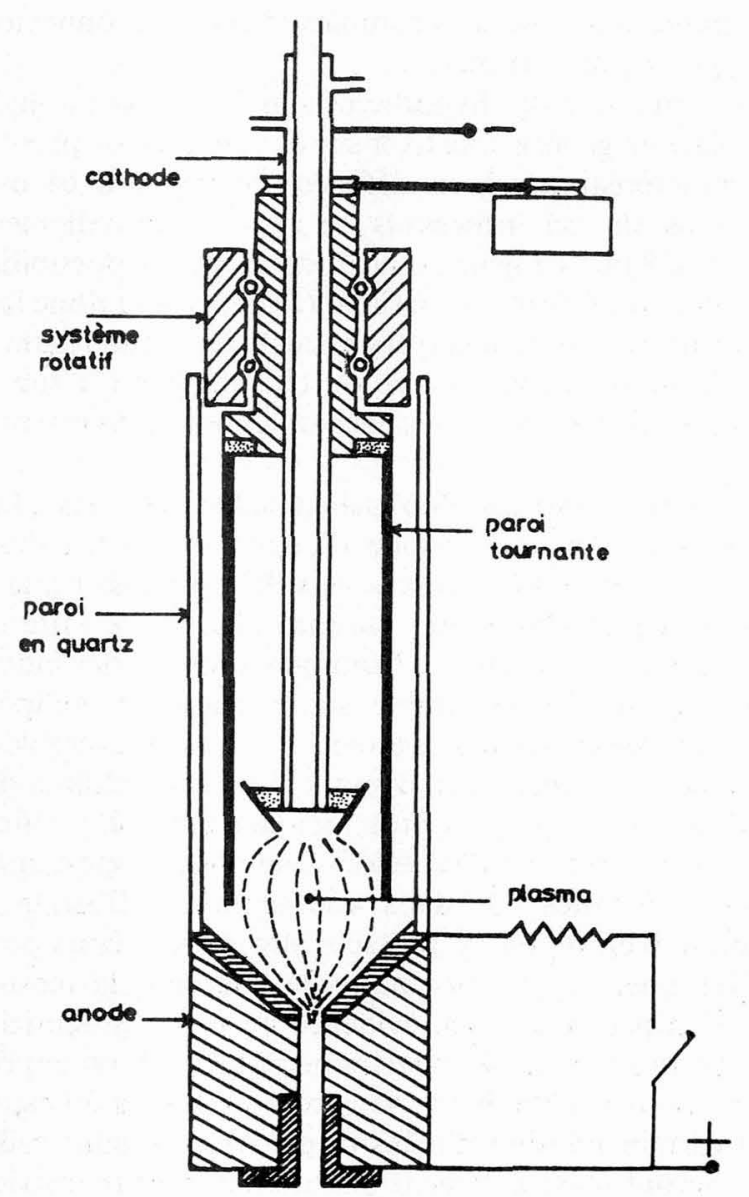

FIG. 14. - Four à cathode et paroi en rotation.

être couplés comme par exemple pour l'arc alternatif de la Westinghouse [210] (Fig. 15),

- établissement d'un lit fluidisé [211]. Par exemple sur la figure 16 nous avons représenté le système développé à Odeillo $[212,213]$ où le plasma est 


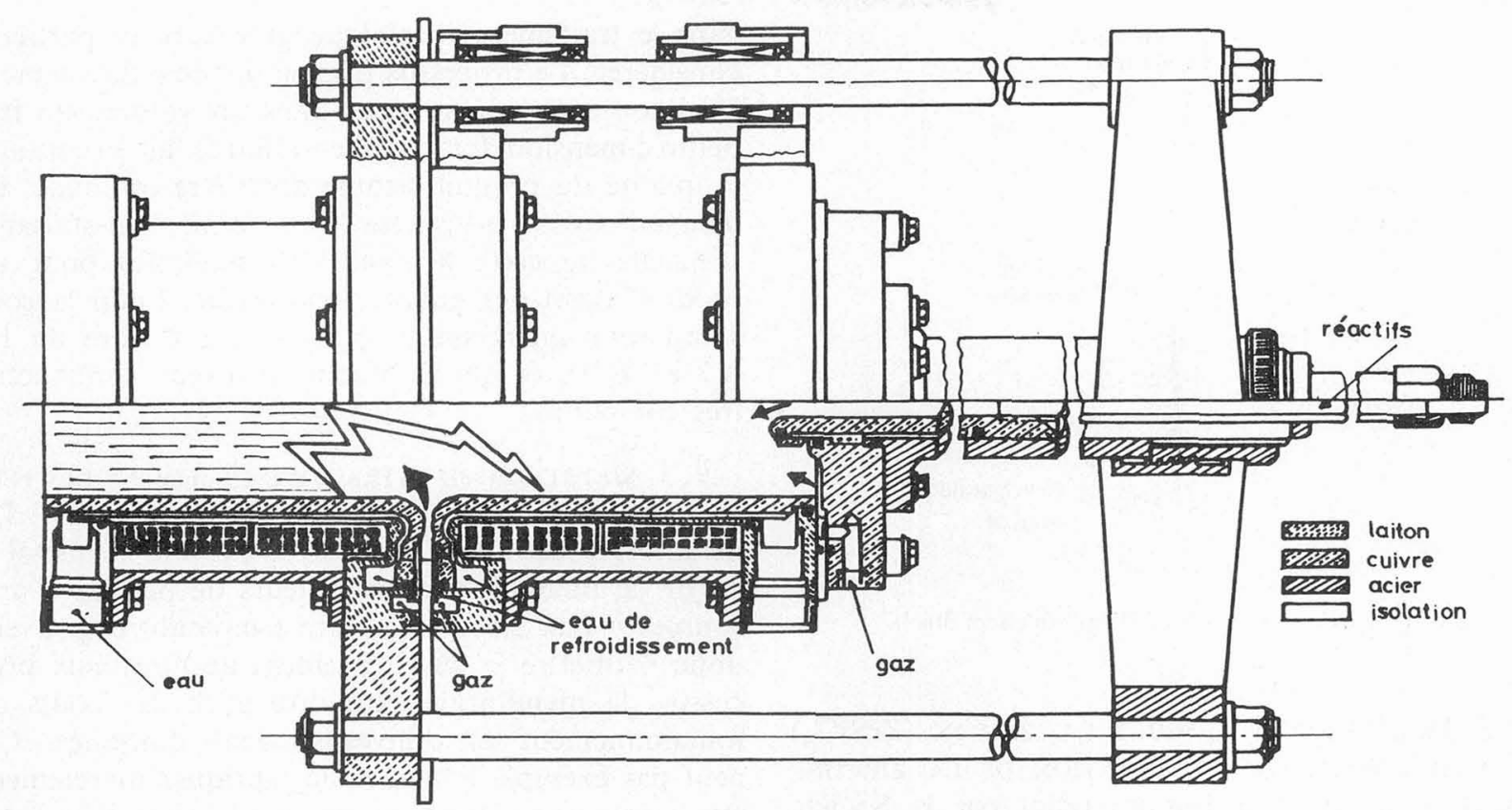

FIG. 15. - Arc auto-stabilité alternatif, Westinghouse.

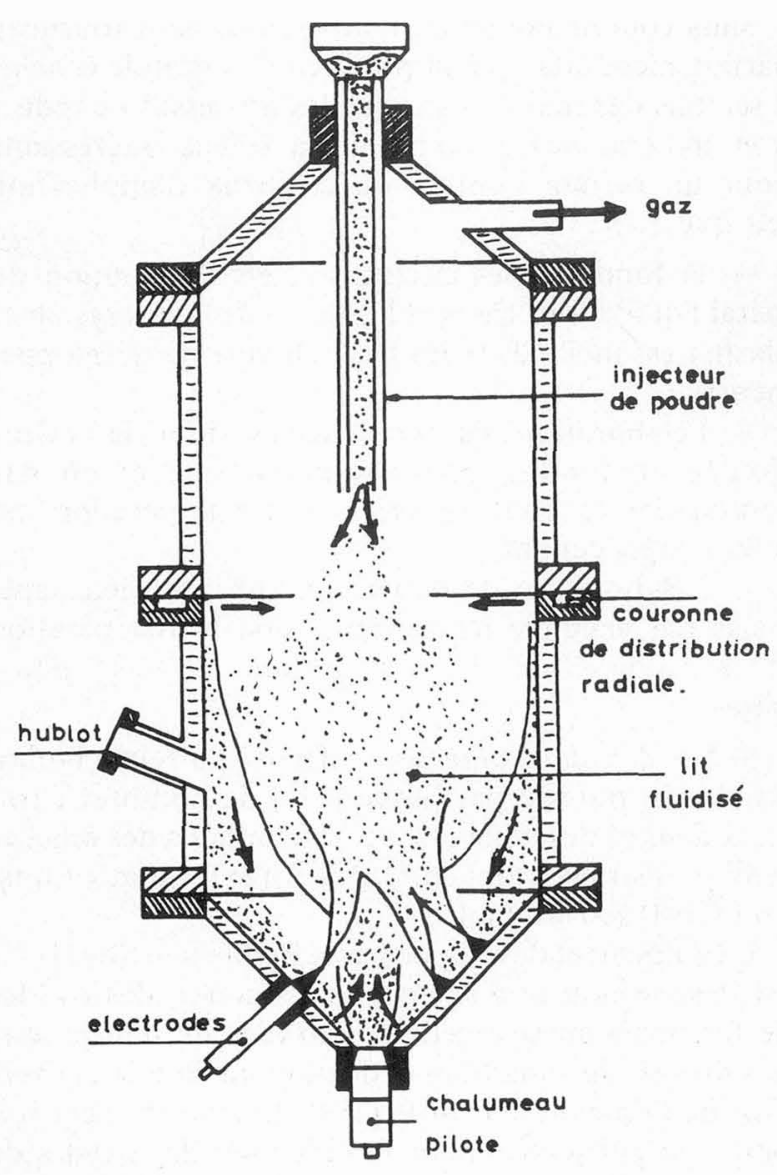

FIG. 16. - Réacteur en lit fluidisé (Odeillo).

produit entre trois électrodes alimentées en courant alternatif triphasé. Les poudres non traitées tombent dans le cône inférieur et sont réintroduites dans le lit fluidisé par l'écoulement de gaz froid du chalumeau pilote,

- établissement d'un film ruisselant [214] lorsqu'il s'agit de traiter des matériaux en fusion (les temps de séjour peuvent dépasser $10 \mathrm{~s}$ ). Par exemple sur la figure 17 nous avons représenté le four de Sayce [215] pour lequel la poudre introduite est centrifugée sur la paroi où elle constitue un film liquide ruisselant. Sur la figure 18 , nous avons représenté le dispositif de la Bethlehem Steel [141] où le film ruisselant sert d'anode. - Naturellement, chaque solution a ses avantages et ses inconvénients et dans tous les cas le réacteur doit être calculé en fonction du problème à traiter.

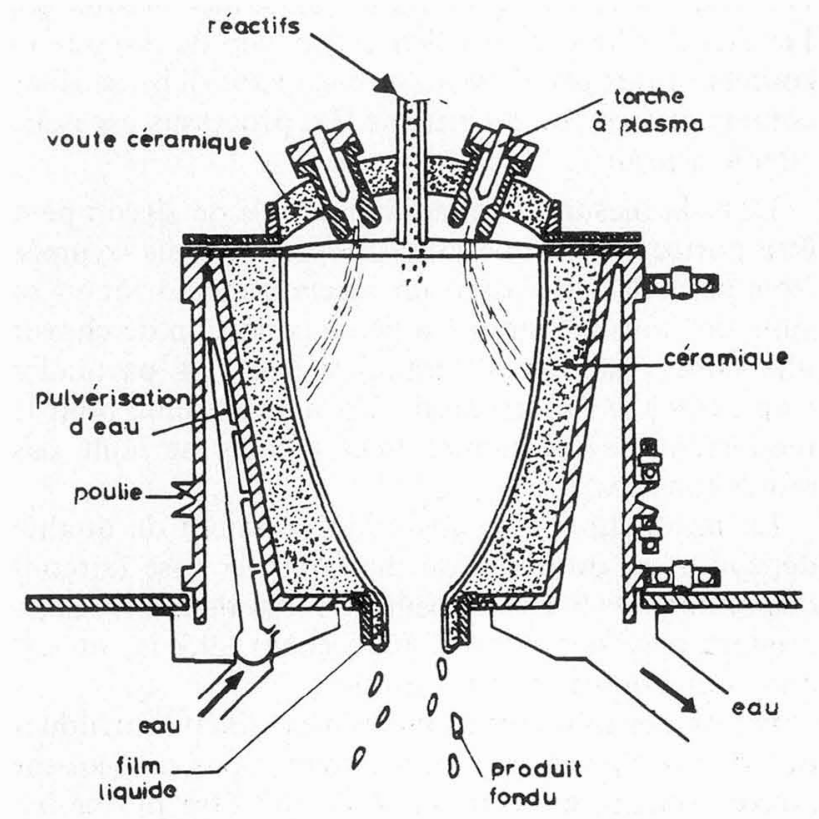

FIG. 17. - Four à plasma centrifuge (Sayce). 


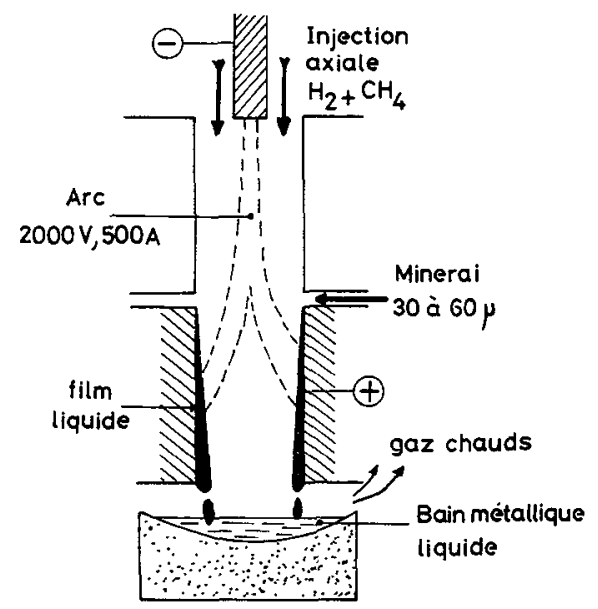

Fig. 18. - Four à arc de 1 MW (Bethlehem Steel).

9.2 TRAITEMENT THERMIQUE DU ZIRCON $\left(\mathrm{ZrSiO}_{4}\right)$ EN PLASMA POUR LA RÉCUPÉRATION DE LA ZIRCONE $\left(\mathrm{ZrO}_{2}\right)$. - Ce procédé mis au point par la Société IONARC à Concorde (NH-USA) [202], consiste en un réacteur de $1 \mathrm{MW}$ (courant continu) destiné à la récupération de $\mathrm{ZrO}_{2}$ en deux étapes :

- passage de sable de zircon $\mathrm{ZrSiO}_{4}$ dans un réacteur fonctionnant suivant le principe de l'effet de Maecker (Fig. 13) (les particules injectées au bout de la cathode sont introduites le long de l'arc grâce à la pression magnétique existant à la pointe de la cathode). Lors de cette opération le silicate de zirconium est décomposé en oxydes de silicium $\mathrm{SiO}_{2}$ et de zirconium $\mathrm{ZrO}_{2}$

- lavage des sphérules par une solution de soude pour éliminer la silice sous forme de silicate de sodium,

- le zircon se dissocie spontanément à $2048 \mathrm{~K}$ et au-delà de $3073 \mathrm{~K}$ tous les composants sont à l'état liquide et le diagramme de phase met en évidence l'existence d'une phase riche en oxyde de zirconium contenant très peu de silice et une phase riche en silice contenant très peu de zircone. Le processus est donc simple a priori.

Dans la mesure où chaque particule de zircon peut être portée à la température nécessaire, puis trempée (trempe de la paroi dans un réacteur cylindrique à la suite de l'arc) la réaction a lieu à condition de choisir une bonne gamme de granulométrie (les particules trop petites se vaporisent de façon désastreuse pour le rendement, les particules trop grosses ne sont pas toutes fondues).

La production d'un matériau (zircone) de qualité dépend aussi du choix du matériau de base (zircon) disponible. Or le zircon peut être issu de nombreuses origines avec des puretés approchant $99,7 \%$, on est donc dans un cas très favorable.

Les processus conventionnels nécessitent l'introduction d'autres produits avec le zircon et par conséquent l'oxyde obtenu est contaminé et doit être purifié, ce que l'on sait faire avec plus ou moins de bonheur sui- vant le traitement thermique qu'a subi la particule considérée. Le processus plasma dissocie directement le zircon sans aucun ajout dans un volume de très petite dimension dont l'alimentation se fait en continu, la qualité du produit semble donc être optimale. La demande des utilisateurs est donc satisfaisante (l'échelle de cette demande est modeste) pour un produit ayant des qualités constantes. Enfin la consommation énergétique totale est de l'ordre de 1,5 à $2 \mathrm{kWh} / \mathrm{kg}$, ce qui la situe à un niveau commercial très compétitif.

9.3 MÉTALluRgIE EXTRACTIVE DU FER ET DES FERROALliages. - 9.3.1 Situation du problème. - La métallurgie extractive est l'art d'extraire le métal à partir de minerai. Les générateurs de plasma à arc, sources à flux élevé et à forte température, peuvent donc permettre le développement de nouveaux processus de métallurgie extractive avec des coûts de fonctionnement et d'investissement diminués. On peut par exemple imaginer de fabriquer directement des aciers par plasma en une seule opération [141] à condition de disposer de minerais finement divisés (30 à $100 \mu \mathrm{m}$ ).

Sans vouloir prétendre remplacer le haut fourneau parfaitement adapté à la production à grande échelle, il semble, dès maintenant, que les processus de réduction minerai de fer par plasma soient intéressants pour un certain nombre de créneaux d'application tels que :

- la fonderie (les circuits actuels d'obtention du métal liquide sont chers et l'implantation des systèmes plasma est facile dans les pays en voie de développement),

- l'élaboration de ferroalliages dont la valeur ajoutée au produit final est importante et où par conséquent le coût énergétique de l'opération est moins préoccupant,

- l'élaboration de matériaux aux propriétés spéciales par exemple les métaux purs, la récupération et la valorisation de sous-produits (boues, poussières...).

9.3.2 Résultats obtenus. - Depuis la revue bibliographique publiée par Sayce [216] de nombreux travaux dans ce domaine ont été développés à des échelles semi-industrielles tant en U.R.S.S. [217] qu'aux EtatsUnis [141] par exemple.

L'équipement développé par la Bethlehem Steel [141] est destiné non seulement à la réduction des oxydes de fer, mais aussi à celle des oxydes de manganèse, de chrome, de vanadium et de niobium et à la conversion de l'ilménite en rutile [218]. Les expériences initiales, en particulier pour la réduction des oxydes de fer, ont été développées à une échelle de $100 \mathrm{~kW}$ et les résultats encourageants obtenus à cette puissance ont conduit à la construction d'une unité pilote de $1 \mathrm{MW}$.

Le générateur schématisé sur la figure 18 comporte une cathode en tungstène gainée par une circulation 
d'hydrogène, l'expansion de l'arc derrière la cathode est limitée par une électrode en cuivre à un potentiel flottant derrière laquelle peut être injecté du méthane. Stabilisé par une paroi cylindrique refroidie, le film coule ensuite dans un creuset chauffé par les gaz plasma s'échappant du réacteur. Les particules trop fines sont entraînées dans le gaz plasma et leur temps de séjour y est généralement trop court pour qu'elles subissent une transformation chimique complète qu'elles achèvent alors dans le creuset. Avec un tel dispositif, on atteint un rendement thermique de $90 \%$ et les temps de résidence des particules d'oxydes à traiter varient d'une seconde à une minute.

TABLEAU IV

\section{Consommation énergétique. Pureté}

\begin{tabular}{|c|c|c|}
\hline $\begin{array}{c}\text { Niveau } \\
\text { de puissance }\end{array}$ & Matériau utilisé & $\begin{array}{l}\text { Consommatio } \\
\text { d'énergie } \\
\text { (kWh/kg) }\end{array}$ \\
\hline - & - & - \\
\hline \multirow[t]{5}{*}{$100 \mathrm{~kW}$} & Ecailles de laminage broyées & 3,5 \\
\hline & Magnétite de Pea Ridge & 3,5 \\
\hline & Hématite de Pea Ridge & 4,8 \\
\hline & Magnétite d'Erié & 5,1 \\
\hline & Poudre fine de Grace & 5,5 \\
\hline \multirow[t]{3}{*}{$1 \mathrm{MW}$} & Hématite de Carol Lake (*) & 3,3 \\
\hline & Magnétite de Grace & 4,6 \\
\hline & Hématite de Pea Ridge & 4,8 \\
\hline
\end{tabular}

$\left(^{*}\right)$ Analyse du fer obtenu à partir de l'Hématite de Carol Lake.

$\begin{array}{ccccc}\mathbf{S} & \mathbf{P} & \mathrm{C} & \mathrm{Si} & \mathrm{Cu} \\ 0,005 \% & 0,001 \% & 0,006 \% & 0,06 \% & 0,007 \%\end{array}$

Le système pilote de $1 \mathrm{MW}$ peut fonctionner en continu et produire plus de 200 kilos de fer fondu par heure. De très nombreux minerais ont été testés (cf. Tableau IV) en utilisant un plasma hydrogèneméthane dont la composition variait de 100 à $65 \%$ d'hydrogène. Aux températures atteintes dans le réacteur, on suppose que le carbone provenant du méthane est entièrement converti en monoxyde de carbone, ce que semble confirmer le bilan énergétique. Les résultats ont été représentés sous forme de l'enthalpie fournie au gaz en fonction de la consommation énergétique nécessaire pour obtenir un kilo de fer (Fig. 19). On constate que le rendement s'améliore

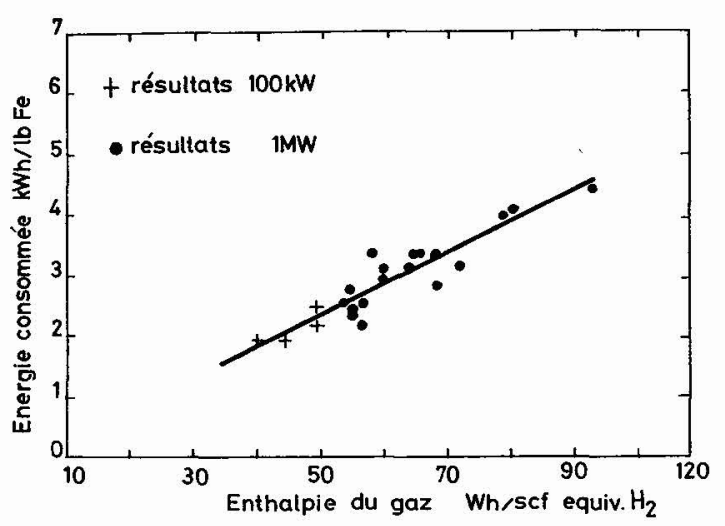

FIG. 19. - Réduction des oxydes de fer : évolution de l'énergie consommée en fonction de l'enthalpie du gaz. lorsque la puissance augmente, ceci est dû à un meilleur rendement thermique du générateur. Le produit fondu est du fer très pur (cf. Tableau IV) et enfin les gaz à la sortie du réacteur (ils sont encore à $2300 \mathrm{~K}$ ) sont récupérés par reforming [218].

Les expériences faites avec le pilote industriel de 1 MW de la Bethlehem Steel Co. ont donc démontré qu'il est parfaitement possible de convertir directement l'oxyde de fer en fer fondu en une seule opération dans un réacteur plasma, toute l'énergie nécessaire étant d'origine électrique. De plus en récupérant les gaz à la sortie par reforming, l'énergie consommée (kWh/kg de fer) est assez voisine du minimum théorique, ce qui est très encourageant pour des développements ultérieurs à une plus grande échelle.

Comme nous l'avons déjà mentionné, la production de ferroalliages est encore plus rentable que celle du fer pur, c'est ainsi que la Bethlehem Steel a mis au point avec le même réacteur un procédé permettant d'obtenir directement du ferrovanadium en consommant une énergie de l'ordre de $8 \mathrm{kWh} / \mathrm{kg}$ (la réduction étant assurée essentiellement par le charbon). Le coût de l'énergie intervient ici pour une part assez faible du coût total (1/6 environ) et si l'on compare ce procédé aux procédés actuels de réduction de $\mathrm{V}_{2} \mathrm{O}_{5}$ par $\mathrm{Si}, \mathrm{Al}$ ou $\mathrm{C}$ par fusion sous vide, ces derniers sont de plus grands consommateurs d'énergie. Nous pouvons également souligner l'étude théorique de Fey [1] pour un système de production de ferrochrome. Dans cette étude il envisage un processus en deux étapes où un minerai de chromite à faible teneur est d'abord pré-réduit par les gaz obtenus à la sortie du réacteur plasma, ce qui permet, à la sortie de ce pré-réducteur, d'éliminer le fer obtenu par exemple par séparation magnétique et d'injecter la chromite à forte teneur dans le réacteur à plasma où elle est réduite en ferrochrome par le gaz naturel (Fig. 20). En suppo-

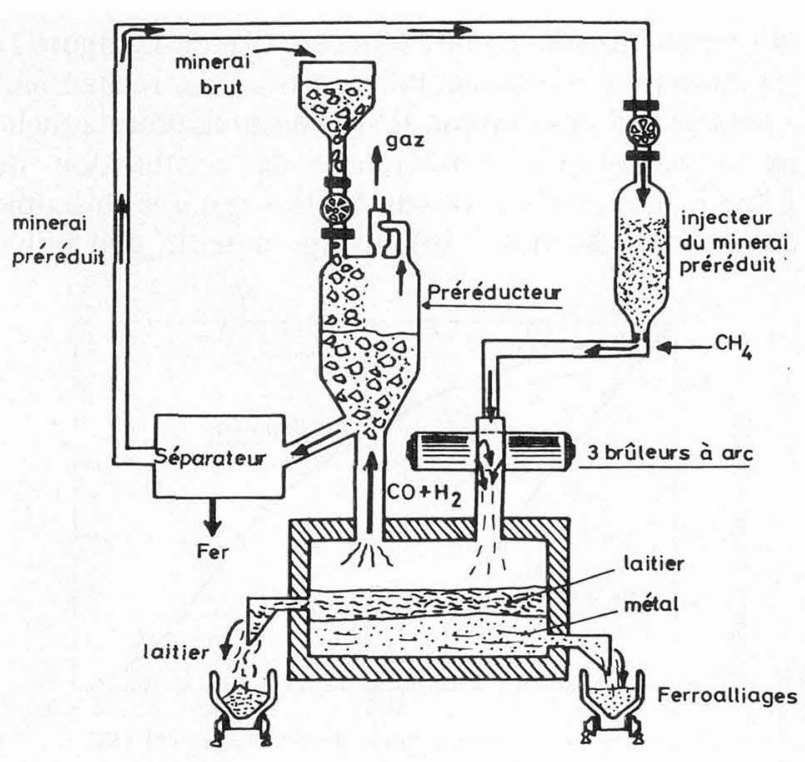

FIG. 20. - Schéma du réacteur de la Westinghouse pour la synthèse des ferrochromes. 
sant que l'énergie théorique nécessaire est de $14 \mathrm{kWh} /$ $\mathrm{kg}$ pour un minerai contenant un rapport chromefer de 1,6 et que le rendement est de $50 \%$ pour une unité produisant 16000 tonnes par an et fonctionnant 8000 heures par an àvec $16 \mathrm{MW}$, il démontre que le coût de production est d'environ $\$ 0,48$ par $\mathrm{kg}$ de métal produit, ce qui, toujours d'après ces calculs, est extrêmement rentable.

9.3.3 Conclusions. - Les réacteurs pilotes à l'échelle d'un mégawatt ont donc parfaitement démontré leurs possibilités d'utilisation industrielle. Toutefois actuellement ils ne sont encore rentables au plan économique que pour des ferroalliages où par exemple pour le ferrovanadium la production nécessaire aux U.S.A. est de 1000 tonnes/an, ce qui peut facilement se faire avec un réacteur d'un MW. Par contre la plupart des systèmes métallurgiques nécessitent une production beaucoup plus élevée, donc un niveau de puissance très supérieur. Par exemple une production d'acier de 250000 tonnes/an nécessiterait un réacteur de $100 \mathrm{MW}$, type de réacteur qui reste à développer (on peut citer les essais de Wright-Patterson à $40 \mathrm{MW}$ [219] et de la NASA (encore sur plans) à $60 \mathrm{MW} \mathrm{[220]).} \mathrm{Il} \mathrm{faut} \mathrm{en} \mathrm{particulier} \mathrm{pour} \mathrm{ce} \mathrm{type} \mathrm{de}$ réacteur améliorer la durée de vie des électrodes pour diminuer le coût de la maintenance et pour tous les réacteurs récupérer l'énergie des gaz à la sortie soit par reforming soit par préréduction. Il n'en demeure pas moins vrai qu'en raison de la conjoncture économique il y a là un débouché potentiel très important dans moins de 10 ans en France et moins de 20 ans aux U.S.A.

9.4 LES FOURS DE REFUSION. - Les réchauffeurs à plasma peuvent être utilisés pour un grand nombre de processus à haute température étant donné que de très nombreux gaz peuvent être portés à température élevée et que l'on peut aussi éviter de nombreux problèmes de contamination rencontrés avec les produits de combustion des réchauffeurs classiques. La figure 21 [1] indique les rendements respectifs d'un réchauffeur à plasma et d'une flamme air-gaz naturel, pour laquelle on a admis une température de combustion de $2233 \mathrm{~K}$. Les produits de combustion ont une enthalpie qui ne dépasse pas $3161 \mathrm{~kJ} / \mathrm{kg}$ et seule une faible

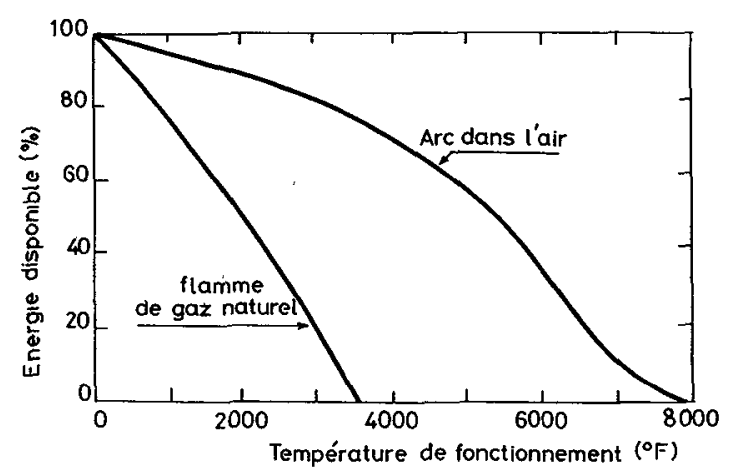

Frg. 21. - Rendements respectifs d'un réchauffeur à plasma et d'une flamme air-gaz naturel. fraction de l'énergie est récupérable suivant la température du four alors qu'un réchauffeur à plasma d'air peut atteindre une enthalpie de $9298 \mathrm{~kJ} / \mathrm{kg}$. Par exemple Fey [1] démontre qu'aux U.S.A. un réchauffeur à plasma est actuellement moins cher qu'un brûleur à méthane si l'électricité ne coûte que 2,4 cents/ $\mathrm{kWh}$ et il suggère d'utiliser de tels réacteurs pour le chauffage des brames.

Signalons également dans le même esprit le four de refusion d'une centaine de $\mathrm{kW}$ mis au point par la société Electrotherm [221, 222] et qui comporte :

- un système de chauffage constitué par trois générateurs à plasma,

- une enceinte refroidie où l'on peut avoir soit une atmosphère contrôlée, soit une pression réduite,

- une lingotière refroidie par circulation d'eau,

- une barre animée d'un mouvement de descente et de rotation.

Les jets de plasma pilote, produits par les trois générateurs (DC), convergent vers la barre et un intense courant alternatif triphasé est établi entre les torches, le point neutre flottant étant constitué par la barre. L'extrémité de celle-ci fond superficiellement et les gouttes obtenues tombent dans le bain. Les pertes thermiques restent importantes mais ce four peut être utilisé pour l'affinage de niobium, du tantale, du titane et autres métaux ou alliages.

Enfin outre le four rotatif à axe vertical de Sayce [215] déjà décrit sommairement (Fig. 17) dont 1'un des principaux problèmes est la tenue thermique de la voûte surchauffée par le rayonnement du plasma, il reste à décrire sommairement le four rotatif à axe horizontal [223, 224]. Ce four comporte :

- deux générateurs à plasma (DC) face à face, - une enveloppe à symétrie cylindrique, refroidie par eau, mobile autour d'un axe horizontal et contenant le matériau à fondre.

La matière préalablement disposée dans le four sous forme divisée est centrifugée et on réalise une superposition de puissance entre les deux générateurs à plasma. Il y a alors fusion du matériau sur une certaine épaisseur, le liquide étant lui-même centrifugé. Pour obtenir une coulée du matériau fondu on diminue la vitesse de rotation et on incline le four.

Avec le four expérimental de Saclay de $130 \mathrm{~kW}$ [224] on a pu ainsi fondre des oxydes réfractaires comme l'alumine, la magnésie et la zircone. Pour des fours de plus grande puissance il reste à résoudre le problème de l'amorçage car les générateurs à plasma ne peuvent être rapprochés et il faut donc que les jets de plasma produits à l'origine chauffent suffisamment le gaz contenu dans la cavité pour que la superposition puisse s'établir, ce qui dépend beaucoup de la conductivité thermique du matériau à traiter constituant la cavité.

9.5 SYNTHÈSE DE L'ACÉTYLÈNE DANS UN ARC ÉLECTRIQUE. - 9.5.1 Position du problème. — L'acéty- 
lène, hydrocarbure gazeux, est principalement utilisé comme intermédiaire dans les chaînes de fabrication chimique (plastiques, peintures à l'eau, matériaux vinyliques, produits d'entretien, aérosols...). Classiquement la synthèse de l'acétylène est réalisée par hydrolyse du carbure de calcium $\mathrm{CaC}_{2}$ ou par oxydation partielle du méthane dans l'oxygène. Durant les dix dernières années ce produit a été progressivement remplacé par l'éthylène essentiellement pour des raisons économiques. Mais la fabrication de ce dernier nécessite beaucoup d'hydrocarbures. Compte tenu de l'évolution de l'espace énergétique que nous avons décrit au début de cet article, on comprend qu'un procédé plasma (arc électrique) utilisant par exemple le charbon puisse éventuellement rendre à l'acétylène la place qu'il occupait voici quelques années.

9.5.2 Procédés utilisant les arcs électriques. Deux méthodes principalement sont applicables :

- la première consiste à craquer le méthane ou divers hydrocarbures dans un arc fonctionnant généralement à l'hydrogène

$$
2 \mathrm{CH}_{4} \stackrel{(\operatorname{arc})}{\longrightarrow} \mathrm{C}_{2} \mathrm{H}_{2}+3 \mathrm{H}_{2}
$$

- la seconde consiste simplement en la synthèse à partir des éléments

$$
2 \mathrm{C}+2 \mathrm{H}_{2} \stackrel{\text { (arc) }}{\longrightarrow} \mathrm{C}_{2} \mathrm{H}_{2} \text {. }
$$

En fait, d'un point de vue économique, le second système devrait s'avérer le plus rentable dans les années à venir.

Remarquons par ailleurs que de récents rapports américains font état de possibilités autres que les deux précédentes. Par exemple un retour au procédé basé sur le carbure de calcium est envisagé au niveau plasma dans la mesure où la synthèse de $\mathrm{CaC}_{2}$ serait possible dans un arc (Massachusetts Institute for Technology, School of Engineering, Cambridge, Massachusetts, par Badour R. F., Howard V. B., Meisner H. P.) à partir du carbonate $\mathrm{CaCO}_{3}$. Par ailleurs la Société AVCO envisage une série d'essais utilisant, en guise de matière première, les déchets de raffinerie plutôt que d'utiliser les hydrocarbures raffinés. La faisabilité de ces nouvelles filières n'a pas encore été vérifiée, d'autres idées peuvent encore apparaître, il semble donc qu'un vaste champ de recherches puisse s'ouvrir dans les années à venir sur ce genre de synthèse. Notons à ce propos que les investigations menées au niveau de $\mathrm{C}_{2} \mathrm{H}_{2}$ peuvent être transposées à d'autres matériaux de synthèse tels que $\mathrm{NO}, \mathrm{HCN}, \ldots$

9.5.3 Le procédé Huels I [208]. — Ce procédé a maintenant plus de dix ans et il a été depuis lors largement amélioré comme nous le verrons par la suite, cependant, nous en donnons ici une brève description car il constitue une bonne image des différents procédés à arc. Le système est représenté sur la figure 22 . L'arc qui peut atteindre une longueur de $1 \mathrm{~m}$ est établi sous $7000 \mathrm{~V}$ et $1150 \mathrm{~A}$ y sont dissipés. Le gaz d'arc est l'hydrogène, le réactif est un hydrocarbure (du méthane à $\mathrm{C}_{4} \mathrm{H}_{10}$ ), ce dernier étant injecté tangentiellement pour stabiliser l'arc. La trempe réactive est réalisée par injection d'hydrocarbures liquides en aval de l'arc, elle est suivie d'une trempe secondaire par pulvérisation d'eau. Les résultats obtenus sur cette configuration ont été rassemblés sur la figure 23. Le graphe porte en abscisse le nombre de carbone qui représente le taux de mélange hydrogène-hydrocarbures. Les chiffres obtenus sont considérés comme satisfaisants pour une première étape. Outre l'acétylène, de nombreux autres hydrocarbures sont récupérés, certains sont des sous-produits du système et commercialisés (éthylène par exemple), les autres sont recyclés dans le four.

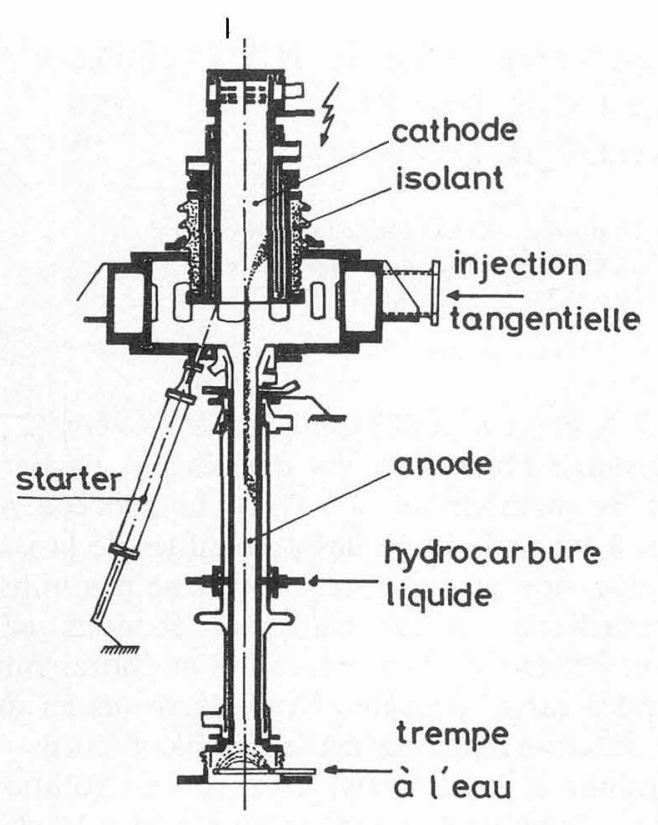

FIG. 22. - Procédé HUELS I.

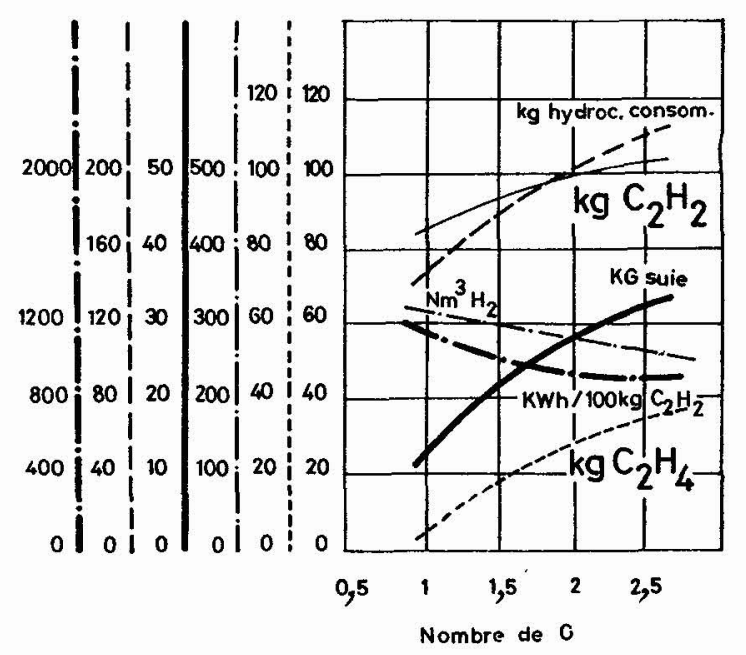

FIG. 23. - Produits obtenus avec un mélange $\mathrm{CH}_{4}-\mathrm{C}_{4} \mathrm{H}_{10}$ pour $1000 \mathrm{kWh}$ d'énergie électrique. 
TABLEAU $\mathrm{V}$

Performances des réacteurs à arc

\begin{tabular}{|c|c|c|c|c|c|}
\hline & $\begin{array}{c}\text { Huels I } \\
-\end{array}$ & Huels II & Hoechst & $\begin{array}{c}\text { Du Pont } \\
\text { de Nemours } \\
-\end{array}$ & $\begin{array}{l}\text { Projet } \\
\text { AVCO (*) } \\
\text { charbon }\end{array}$ \\
\hline Tension & 7100 & 7100 & 1400 & 3500 & 8000 \\
\hline Courant & 1200 & 1200 & $\begin{array}{c}4200 \\
3 \text { phases }\end{array}$ & 3100 & 1250 \\
\hline Pression $(\mathrm{dtm})$ & 1 & 1 & 1 & 0,5 & 0,5 \\
\hline Hydrocarbure (ou char) $\left(^{*}\right)$ arc & $3100(\mathrm{lb} / \mathrm{h})$ & 0 & 0 & 4200 & 8000 \\
\hline Hydroc. (char) $\left({ }^{*}\right)$ après l'arc & $2200(\mathrm{lb} / \mathrm{h})$ & 7000 & 4500 & 0 & 2000 \\
\hline Débit $\mathrm{H}_{2}$ (recyclé) (SCFM) & 2150 & 1400 & 1470 & 3200 & 3000 \\
\hline Enthalpie $\mathrm{H}_{2}(\mathrm{~kW} / \mathrm{SCFM})$ & 4,0 & 6,1 & 6,8 & 3,4 & 3,5 \\
\hline $\mathrm{C}_{2} \mathrm{H}_{2}$ produit $(\mathrm{lb} / \mathrm{hr})$ & 1860 & 1860 & 2200 & 2700 & 3000 \\
\hline Energie consom. $\left(\mathrm{kWh} / \mathrm{lbC}_{2} \mathrm{H}_{2}\right)$ & 4,6 & 4,6 & 4,6 & 3,0 & 4,0 \\
\hline Concent. $\mathrm{C}_{2} \mathrm{H}_{2}(\mathrm{~mol} \%)$ & 15,9 & 15,0 & 14,5 & 18 & 15,5 \\
\hline Concent. $\mathrm{C}_{2} \mathrm{H}_{4}(\mathrm{~mol} \%)$ & 7,1 & 6,4 & 6,5 & 3,0 & $(* *)$ \\
\hline
\end{tabular}

9.5.4 Procédé AVCO utilisant le charbon [225]. Le système Huels $I$ et ses dérivés fait partie de la première méthode (cf. $\S 9.5 .2$ ). Le procédé AVCO quant à lui réalise une des possibilités de la seconde méthode. Son gros avantage est de ne pas utiliser les hydrocarbures, et le rendement économique s'en ressent beaucoup. Trois réacteurs au moins ont déjà été testés sur ce procédé. Nous décrirons ici un système intermédiaire de cet ensemble d'essais. L'arc fonctionne à l'hydrogène, il est mis en rotation par l'action d'un champ magnétique extérieur. Le charbon est injecté à divers niveaux dans l'arc (en amont, au centre et en aval) à une cadence de l'ordre de $450 \mathrm{~g} / \mathrm{min}$. dans chaque section. Le débit d'hydrogène est de $680 \mathrm{l} / \mathrm{min}$. avec une puissance d'arc comprise entre 80 et $100 \mathrm{~kW}$. L'énergie consommée est de $4 \mathrm{~kW} / \mathrm{lb}$ de $\mathrm{C}_{2} \mathrm{H}_{2}$, rendement considéré comme optimal par AVCO. Présentement cette même société compte élargir, dans un proche avenir, son procédé à l'échelle des $10 \mathrm{MW}$.

9.5.5 Autres systèmes. - Les deux procédés que nous venons de décrire ne sont pas les seuls, on peut citer par ailleurs les procédés Huels II, Hoechst A. G., Du Pont de Nemours, ainsi qu'un procédé roumain et russe [226-230]. Le tableau $\mathrm{V}$ résume les performances comparées des principaux procédés. A l'échelle du laboratoire le problème de la synthèse de $\mathrm{C}_{2} \mathrm{H}_{2}$ dans les jets de plasma (d'arc ou HF) a été et est encore étudié de façon plus fondamentale. On s'attache à la compréhension des réactions élémentaires et phéno- mènes de trempe divers. L'aboutissement de ces recherches n'est pas encore totalement réalisé, mais on a pu mettre en évidence l'action déterminante de certains intermédiaires tels que $\mathrm{CH}$ ou $\mathrm{C}_{2} \mathrm{H}$ [231-234].

10. Conclusion. - Longtemps abordée d'un point de vue purement fondamental, la chimie des plasmas voit actuellement son champ d'action s'élargir de façon considérable jusqu'au niveau des applications industrielles. Ce phénomène que l'on explique du point de vue économique par une évolution rapide des ressources et des besoins énergétiques est également dû à une progression importante des connaissances en Physique et Chimie des Plasmas. C'est pour cette raison que l'on peut à l'heure actuelle effectuer des calculs de bilans industriels à l'aide des données de la Physique et de la Chimie fondamentales. Les recherches à caractère technologique portant sur les réacteurs industriels évoluent rapidement grâce aux études expérimentales de laboratoire qui permettent à présent de contrồler de façon précise la faisabilité des procédés. La mise en place de chaînes de fabrication (synthèse de l'acétylène et de l'oxyde d'azote, synthèse de substances organiques, traitement de surface, etc...) montre l'intérêt qu'il faut porter aux systèmes plasma tant au niveau du laboratoire de recherche qu'au niveau des débouchés industriels. De multiples études peuvent encore être abordées dans ce domaine qui bien que largement entamé demeure encore probablement plein de ressources inexploitées. 


\section{Bibliographie}

[1] FEY, G., Electric arc heater for the process industries, National Industrial Electric Heating Conference Cincinnati, Ohio USA, Feb. 9-12, 1976.

[2] Edstrom, J. S., Petrochem. Industr. 2 (1904) 399.

[3] Morssan, H., Le Four électrique (Paris Steinheit) 1897.

[4] Hare, R., Trans. Am. Phil. Soc. 7 (1840) 53. Voir aussi :

KUHN, W. E. ed., Arcs in inert atmosphere and vacuum (New York Wiley) 1956.

[5] CurTis, H. A., Fixed Nitrogen. New York : Chem. Catalog IV (1932).

[6] ShEER, C., Korman, S., Arcs in inert atmosphere and vacuum (Kuhn, W. E. ed.) (Wiley New York) 1956, p. 180.

[7] JoHn et BADE, W. S., Am. Rocket. Soc. J4 (1961) 31.

[8] Skifstad, J. G., Summary of published litterature concerned with electric arc phenomena pertinent to plasma jet devices. Jet propulsion center Rept., Perdue Univ., Lafayette Indiana (1962).

[9] Reed, T. B., Proc. Natl. Electro conf. XIX National electronics conferences Inc., Chicago Illinois (1963) 654.

[10] SheER, C., Plasma technology (Wiley New York) 1967.

[11] Theory and Application of Radio. Frequency Heating Ed. : (Van Nostrand D. CO, N. Y.) 1947.

[12] Leprince-Ringuet, F., Collongues, R., Bull. Soc. Chim. $6(1962) 522$.

[13] Llewellyn-Jones, F., The glow discharge, Ed. : Methuen London 1966.

[14] Besson, A., Comptes Rendus 152 (1911) 1850.

[15] OuCH, K., J. Electrochem. Soc. Japan 17 (1949) 285.

[16] OuchI, K., J. Electrochem. Soc. Japan 20 (1952) 164, 168, 378.

[17] Stokes, Correa, J. J., Streng, L. et Leutner, R., Am. Inst. Chem. Eng. 11 (1965) 377.

[18] Grosse, A. V., Leutner, H. W. et Stokes, C. S., Plasma Jet Chemistry, 1st Ann. Rept. Pa. for Office of Naval Research Contract $\mathrm{n}^{0}$ NONR 3085 (02) Task $\mathrm{n}^{\circ}$ NR 052429 (1961).

[19] Leutner, H. W., Ind. Eng. Chem. Proc. Design Develop. 1 (1962) 166.

[20] Leutner, H. W., Ind. Eng. Chem., Proc. Design Develop. 2 (1963) $4,315$.

[21] Leutner, H. W., Ind. Eng. Chem., Proc. Design Develop. 1 (1962) 167.

[22] BURK, R. E., E. I. du Pont de Nemours Co. Inc., Wilmington, USA, DBP 1038 535, U.S. Prior. (1955).

[23] Farlow, M. W., Muetterties, E. L., Us. Pat. 2709190 (1955); Us. Pat. 2725410 (1955).

[24] Farlow, M. W., Pat. 2081245 (1963).

[25] Farlow, M. W., Muetrerties, E. L., Us. Pat. 2981761 (1961).

[26] Farlow, M. W., Joyce, R. M., Us. Pat. 2709183 (1955).

[27] Bronfin, B. R., Hazlett, R. N., 57th Ann. Meeting A.I. Ch. E. Boston U.S.A. (1964).

[28] LipsCOMB, R. D., Us. Pat. 3043662 (1962).

[29] LipsComb, R. D., SMith, W. C., US. Pat. 3008798 (1961).

[30] Freeman, M. P., Skrivan, J. F., Meet. of the Amer. Inst. of chem. Engineers, N-Y USA (1961).

[31] Arkless, K., Ceaver D., British Titan Products. Co. Ltd. DAS 1226082 (1966) (Prior Brit. 1962).

[32] AMrRICAN CYNAMid. CO. N.Y. U.S.A. Appl. 6605509 (1966) U.S. Appl. (1965).

[33] Korman, S., Sheer, C., Reduction of $\mathrm{MgO}$ in the High Intensity Arc, KLX-10110, Vitro Lab. Tech. Note $\mathrm{n}^{\mathrm{o}} 6$ (1958).

[34] Harris, V., Holmaren, J., Korman, S., Sheer, C., J. Electrochem. Soc. 106 (1959) 874.

[35] Kusch, H. J., Chem. Ing. Techn. 35 (1963) 448.

[36] Stokes, C. S., Cahill, J. A., Correa, J. J., Grosse, Final Rept. Air Force Office of Scientific Res., Grant (1965) 62-196.
[37] SheER. C.. Korman, The high intensity are in process chemistry, Proc. Symp., Electrothermics and Metallurgy Division, Electro-chemical Society, San Francisco (1956).

[38] BosCH, F. M., DE VYNCK, Silic. Indust. 27 (1962) 587.

[39] Leprince-Ringuet, F., Lejus, A. M., Collongues, R., Comptes Rendus 258 (1964) 221.

[40] Leprince-Ringuet, F., Ann. Chim. 2 (1967) 297.

[41] STOKeS, C. S., Correa, J. J., Final Rept for Glidden co. (1964).

[42] Stokes, C. S., Cahill, J. A., Final Rept. Air Force Office of Scientific Res. (1965) Grant 775-65, U.S.A.

[43] Tirche, M., Butti, C., Meth. Phys. Anal. 4 (1968) 379.

[44] Stokes, C. S., KNIPE, W. W., Ind. Eng. Chem. 52 (1960) 287.

[45] Harnisch, H., Heymer, G., Schallus, E., Chem. Ing. Techn. 35 (1963) 7.

[46] Harnisch, H., Heymer, G., Schallus, E., Angew. Chem. 2 (1963) 238.

[47] HeYmer, G., HaRnISCh, H. (Knapsack AG), DBP 1160831 (1964).

[48] HeYMer, G., HaRnisCh, H. (Knapsack AG), DBP 1193485 (1966).

[49] Becherescu, D., Stud. Cerc. Chim. Roman. 16 (1968) 11, 879.

[50] Matsumoto, O., HaYakaWA, Y., Denki Kagaku 34 (1966) 775.

[51] Matsumoto, O., Denki Kagaku 34 (1966) 872.

[52] Matsumoto, O., Denki Kagaku 35 (1967) 488.

[53] Matsumoto, O., Denki Kagaku 35 (1967) 625.

[54] Matsumoto, O., Denki Kagaku 35 (1967) 697.

[55] Marsumoro, O., Denki Kagaku 35 (1967) 870.

[56] Матsumoto, O., Kinzoku (J. Metals) 38 (1968) 15, 69.

[57] Matsumoto, O., Shirato, Y., MiYaZaKI, M., Denki Kagaku 36 (1968) 41.

[58] Matsumoto, O., Shirato, Y., Denki Kagaku 36 (1968) 519.

[59] HAYASH, Y., OKADA, K., KoIDE, S., Yogyo Kyokaï Zasshi 76 (1968) 307, 877.

[60] KoIDE, S., OKaDa, K., HAYASHI, Y., Seikei Daigaku Kogakubu Kogaru Kokoku 5 (1967) 376.

[61] Venugopalan, Plasma chemistry. An introduction, chap. XI, vol. II dans Reactions under Plasma conditions ed. par Venugopalan (Wiley Interscience) 1965.

[62] ReED, T. B., Plasmas for high Temperature Chemistry dans Advances in high temperature chemistry, Ed. L. Eyring.

[63] Polak, L. S., Chemical Process in Low Temperature Plasma (1966) : Pure Appl. Chem. 13 (1966) 345.

[64] Freeman, M. P., Chemical Research in Streaming Thermal Plasmas dans Adv, in High Temp. Chem. (1969).

[65] Bell, A., Fondamentals of Plasma Chemistry, p. 1 in : Techniques and applications of plasma chemistry, ed. : (John Wiley and Sons, N.Y.) 1974.

[66] Baddour, R. F., Timmins, R. S., The application of plasmas to chemical processing (Pergamon Press) New-York (1967).

[67] Papoular, R., Phénomènes électriques dans les gaz. Monographie Dunod, Paris (1963).

[68] Von Engel, A., Ionized gases (Clarendon Press, London) (1957).

[69] Rose, D., Brown, S., Phys. Rev. 98 (1955) 310.

[70] MASSEY et al., J. Appl. Phys. 36 (1965) 1790.

[71] Golant, V. E., Izv. Akad. Nauk. SSSR, Ser. Fiz. 23 (1959) 952.

[72] REED, T. B., J. Appl. Phys. 32 (1961) 821 et 34 (1963) 2266 et 34 (1963) 3146.

[73] ReID, J. W., High Pressure Arc Jets, Paper 61.WA.246. Meeting of American Soc. of Mec. Eng. New York, Nov. (1961).

[74] RAEZER et al., Application of DC Plasma Arc Heating to Hypersonic propulsion testing, J. Space and Rockets 1 (1964) 2.

[75] WINCLER et al., AGARD Specialist Meeting Sept. 1964, Bruxelles. 
[76] Mayo et al., NaSA, T. N., D 2032 Nov. (1963).

[77] EschenBaCH, R. C., WADD, T. R., 61, 100, juillet (1961).

[78] SARlitto, R. J., Tech. Doc. Rep. RTD-TDR-63-4055. Speedway Research Laboratory, Indianapolis (1964).

[79] BrownIng, J., POOLE-AGARD Special Meeting Belgique Sept. (1964).

[80] SHEPAHARD et al., AGARD Special Meeting Belgium (1964).

[81] MARlotte et al., AGARD Special Meeting Belgium sept. (1964).

[82] CANn et BuHler, AGARD Special Meeting Belgium, sept. (1964).

[83] STine, In temperature technology : Proc. of Int. Symp. on High Temp. Techn. A silomar calif. (1964) Buharwoth Washington.

[84] Skifstad, Ph. D Thesis Purdue University, janv. (1964).

[85] NoRTON, MURTHY, Aerospace Research Laboratory ARL Report 65-54, mars (1965).

[86] Fauchais, P., Thèse Doctorat ès Sciences, Poitiers (1968)

[87] FAUCHAIs, P., Les Chalumeaux à Plasma à Arc Electrique dans Les Hautes Températures et leurs utilisations en physique et en chimie. Tome 1 (Masson \& Cie) 1973.

[88] Browning, J., Techniques for producing plasma jets in Dynamics of conducting gases proceedings of the third biennal gaz dynamics symposium. pp. 126-138. (Northwestern University. Press) 1960.

[89] Schoumaker, H. R. P., Fours à chauffage plasma dans Conference proceedings of International Round Table on Study and Applications of Transport Phenomena in Thermal Plasmas. Laboratoire des Ultra-Réfractaires du C.N.R.S., Odeillo sept. (1975).

[90] Schoumaker, H. R. P., Four d̀ plasma triphasé. Publications Electrotherm. Communication au C.B.E.E. Réunion du 30 mars (1971).

[91] Boner, C., Les générateurs de plasma à arc gainé, High Temperatures, High Pressures 3 (1971) 333.

[92] Bonet, C., Lamos, J., Foex, M., Ecoulements permanents de plasma de grande puissance, Entropie 34-35 (1970) 36.

[93] Westinghouse, brochure, From Westinghouse... a new look at the world of plasma chemistry.

[94] SuHR, H., XIe Inter. Conf. on Phenomena in Ionized Gazes (1973). Invited papers, Prague, Tchekoslovaquie.

[95] EMmons, H. W., Recent development in plasma heat transfer (W. Ibele ed.) (Academic Press. New York) 1963.

[96] Timmins, R. S. et Amman, P. R., A.I. Ch. E.J. 12 (1966) 956.

[97] Gladisch, H., Hydro. Proc. \& Pet. Ref. 41 (1962) 6.

[98] Meubus, P., J. Electrochem. 122 (1975) 298.

[99] Amouroux, J., Synthèse et processus chimiques dans un plasma inductif, p. 113 dans les Hautes Températures, Ed. : (Masson, Paris) 1973.

[100] Vursel, F., Polak, L., Plasma Chemical Processing, ch. 16, T. II, in Plasma Chemistry, Ed. : Venugopalan (Wiley Interscience) 1974.

[101] KIRK, K. W., Applications of plasma technology to the fabrication of semi-conductor devices, p. 347 in : Techniques and applications of plasma chemistry, Ed. : (John Wiley, N.Y.) 1974.

[102] Hudis, M., Plasma Treatment of Solid Materials, p. 113 in Techniques and applications of plasma chemistry, Ed. : John Wiley, N.Y. 1974.

[103] Millard, M., Synthesis of organic polymer films in plasma, p. 177 in Techniques and applications of plasma chemistry, Ed : (John Wiley, N.Y.) 1974.

[104] Kondratiev, V. N., Nikitin, E. E., Talrose, V. L., in Nizkotemperaturnaya Plasma (Low-Temperature Plasma, edited by A. E. Scheindlin, Moscow, 1967).

[105] MCDaniel, E. W., Collision Phenomena in Ionized Gases (New York, Wiley) 1964, chap. 11.

[106] Delcroix, J. L., Propriétés Physico-chimiques des gaz simples, Ecole Internationale d'Eté des Houches, août (1976).

[107] Delcroix, J. L., Physique des Plasmas (Dunod) Tome I et II.

[108] Delcroix, J. L., Matos Ferreira, C., RiCARD, A., Metastables atomiques et moléculaires dans les décharges électriques. Rapport L.P. 142, octobre 1973. Laboratoire de Physique des Plasmas. Université de Paris Sud, Centre d'Orsay.

[109] Gilardini, A. L., Low Energy Electron Collisions in Gases Swarns and plasma methods applied to their study. Ed. : (Wiley interscience N.Y.) 1972.

[110] MCDANIEL, E. W., Collision Phenomena in Ionized Gases (Wiley, New York) 1964.

[111] McDaniel, E. W., Cermak, V., Dalgarno, A., Ferguson, E. E., Friedman, L., Ion-Molecule Reactions (WileyInterscience, New York) 1970.

[112] Chapman, S., Cowling, T. G., The Mathematical Theory of Nonuniform Gases (Cambridge) 1952, chap. 18, p. 7.

[113] SMIT, J. A., Physica 3 (1936) 543.

[114] Nikitin, E. E., Sovremennye Teorii Termicheskogo Raspada i Isomerisatsii Molekul $v$ Gasovoy Fase, Moscow : Id-vo Nauka (1964). (Theory of thermally Induced Gas-Phase Reactions, Bloomington, Ind. : Indiana University.

115] BELL, A. T., Fundamentals of Plasma Chemistry in Techniques and Applications of Plasma Chemistry, ed. J. R. Hollahan et Bell A. T. (Wiley-Interscience, N.Y.) 1974.

[116] Polak, L., Some principles of non equilibrium plasma chemical reaction kinetics, ch. 13, p. 141, T. 2, Plasma Chemistry, ed. Venugopalan (Wiley-Interscience, N.Y.) 1971.

[117] Catherinot, A., Sy, A., Z. Naturforsch. 30a (1975) 1143.

[118] Shaw, J. F., Mitchner, M., Kruger, C. H., Phys. Fluids 13 (1970) 325

[119] Cacciatore, M., Capitelli, M., Z. Naturforsch. 31a (1976) 362.

[120] Dumas, J. L., Bull. Soc. Chim. de France 5 (1976) 658.

[121] BELL, A. T., Techniques and applications of plasma chemistry, ed. J. R. Hollahan et A. T. Bell (Wiley-Interscience N.Y.) 1974.

[122] Plooster, Reed, T. B., J. Chem. Phys. (1959) 3166.

[123] Baddour, R. F., Blanchet, J. L., Ind. Ing. Chem. Process. Design Development 3 (1964) 258.

[124] Baddour, R. F., Bronfin, B. R., Ind. Chem. Eng. Process. Design. Dev. 4 (1965) 162.

[125] Marinowski, G. W., Phillips, R. C., Phillips, T. R., HeESTER, N. K., Ind. Eng. Chem. Fundamantals 1 (1962) 52.

[126] Bronfin, B. R., FreEman, M. P., Distefana, V. N., HazletT, R. N., Thermochemical equilibrium in a carbon, hydrogen, nitrogen system at very high temperature. 15th Chem. Inst. of Can. Chem. Eng. Conf. Université Laval, Quebec City, Quebec, oct. (1965).

[127] KRIEGER, F. J., Rand Corp. mars (1971), R, 660, DASA, 2624

[128] KrIEGER, F. J., Rand Corp. oct. (1969) Memorandum RM, 3326, 3, PR.

[129] KrIEGER, F. J., Rand Corp. avr. (1970) Memorandum RM, 6295, PR.

[130] Grimaud, A., Thèse de $3^{e}$ cycle, UER des Sciences, Université de Limoges (1973).

[131] Bourdin, E., Thèse de $3^{\mathrm{e}}$ cycle, UER des Sciences, Université de Limoges, mars (1976).

[132] STOREY, S. H., VAN ZEGEREN, F., The computation of chemical equilibria (Cambridge Univ. Press.) 1970.

[133] Brinckley, S. R., J. Chem. Phys. 9 (1946) 14.

[134] Brinckley, S. R., J. Chem. Phys. 2 (1947) 15.

[135] ARIs, G., Arch. Rat. Mech. and Anal. 19 (1965) 81.

[136] Huff, Gordon, Morrel, NACA Report (1951) (voir également 131,132 )

[137] Landau, L., Lifchitz, E., Physique statistique, Mir, Moscou (1967).

[138] Fauchais, P., Baronnet, J. M., Bayard, S., Rev. Int. Hautes Temp. et Réfrac. 12 (1975) 221.

[139] Baddour, R. F., Blanchet, J. L., Ind. Eng. Chem. Proc. Des. Dev. 3 (1964) 258.

[140] Bourdin, E., Fauchais, P., Grimaud, A., Formation of Aluminium and Silicon nitrides by mean of nitrogen plasma jets, I.U.P.A.C. Symposium on plasma chemistry, Rome 18-23 septembre (1975).

[141] Gold, R. G., Sandall, W. R., Cheplick, P. G., McRae, 
D. R., Plasma Reduction of Iron Oxide with hydrogen and natural gas at $100 \mathrm{~kW}$ and one megawatt, International Round Table on Study and application of transport phenomena in thermal plasmas, I.U.P.A.C. Odeillo 12-16 septembre (1975) proceedings.

[142] Hirschefelder, J. O., CurTiss, C. F., Bird, R. B., Molecular Theory of Gases and Liquids (J. Wiley and Sons, Inc., New York) 2nd edition 1974.

[143] Chapman, S., Cowling, T., The Mathematical Theory of Non-Uniform Gases (Cambridge Univ. Press, New York) 1952.

[144] Mason, E. A., Vandersuice, J. T., Yos J. M., Phys. Fluids 2 (1959) 688.

[145] Nyeland, C., Mason, E. A., Phys. Fluids 10 (1967) 985.

[146] ATHYE, W. F., A critical Evaluation of Methods for Calculating Transport Coefficients of Partially and Fully Ionized Gases, NASA TN, ND-2611 (1965).

[147] Devoto, R. S., Phys. Fluids 9 (1966) 1230.

[148] Devoto, R. S., Phys. Fluids 10 (1967) 2105.

[149] Devoto, R. S., LI, C. P., J. Plasma Phys. 2 (1968) 17.

[150] Devoto, R. S., A.I.A.A. Journal 7 (1969) 12.

[151] Devoto, R. S., Phys. Fluids 16 (1973) 616.

[152] Freeman, C. N., The Thermochemistry of Non-equilibrium Viscous Radiating Blunt Body Layers in $\mathrm{CO}_{2}-\mathrm{N}_{2}$ atmospheres. Ph. D. Thesis, Purdue Université (1969).

[153] Butler, J. N., Brokaw, R. S., J. Chem. Phys. 26 (1957) 1636.

[154] GoRsE, C., Thèse de 3e cycle, Limoges, septembre (1975).

[155] BonNefor, C., Thèse de $3 e$ cycle, Limoges, septembre (1975).

[156] Sandler, S. I., Mason, E. A., Phys. Fluids 12 (1969) 71.

[157] Capitelli, M., Gorse, C., Fauchais, P., J. Chim. Phys. 7-8 (1976) 755.

[158] Marinowski, G. W., Phillips, R. C., Phillips, T. R., Hiester, N. K., Ind. Eng. Chem. Fundamentals 1 (1962) 52.

[159] Baddour, R. F., Bl.AnChet, J. L., Ind. Ing. Chem. Process. Design. Develop. 3 (1964) 258.

[160] Anderson, J. E., Case, L. K., Ind. Eng. Chem. Process. Design. Develop. 1 (1962) 161.

[161] Stokes, C. S., Streng, L. A., Ind. Eng. Chem. Process. Design. Dev. 4 (1965) 36.

[162] Gladise, H., Hydrocarbon. Process. Petrol. Refiner 41 (1962) 159.

[163] Grey, J., JACOBS, P. F., A.I.A.A. J. 2 (1964) 433.

[164] KLINE, S. J., Similitude and approximation Theory (McGrawHill, New York) 1965.

[165] Buckingham, E., E. Phys. Rev. 4 (1914) 345.

[166] VAN DrIEST, E., J. Appl. Mech. Trans. ASME A 34 (1946) 124.

[167] Langhaur, H., Dimensional Analysis and Theory of Models (John Wiley and Sons, Inc., New York) 1958.

[168] Huntley, H. E., Dimensional Analysis (McDonald and Company, London) 1953.

[169] Bird, R. B., Steward, W. E., Lightfoot, E. N., Transport phenomena (John Wiley and Sons, New York) 1960.

[170] Lighfoot, E. N., Estimation of heat and Mass transfer rates. Lectures in Transport phenomena. Edited by R. B. Bird and al., 345 EAST 47 Street New York 10017.

[171] Topham, D. R., J. Phys. D : Appl. Phys. 5 (1972) 533.

[172] WORSOE, P. M., SCHMIDT, Finite difference Solution for laminar flow of gas in a tube at a High Heating Rate, Stanford University, Dept. of Mechanical Engineering, Technical Report No SU-247-8, november (1964).

[173] Schlichting, H., Boundary-Layer Theory (Sixth Ed.) (Mc Graw-Hill, New York) 1968.

[174] Gross, B., Grycz, B., MikLossy, K., Plasma Technology (Eng. Trad.) (ILIFFE Books LTD London) 1968.

[175] NorTon, D. J., MURTHY, S. N. B., An Investigation of Energy Transfer in a tube Arc Heater, A.R.L., 65-54, March (1965).

[176] Paulson, R. F., Pfender, E., IEeE Transactions of Plasma Science 3 (1973) 256.

[177] ShiH, K. T., PFender, E., IBele, W. E., ECKert, E. R., A.I.A.A. J. 6 (1968) 1021.

[178] ShiH, K. T., PFender, E., A.I.A.A. J. 8 (1970) 786.

[179] BOSE, T. K., PFENDER, E., A.I.A.A. J. 7 (1969) 832.
[180] Wutzke, S. A., Pfender, E., Eckert, E. R. G., A.I.A.A. J. 6 (1968) 903.

[181] BOWER, W. W., INCROPERA, F. P., Warme-und -Stoffubertragung 2 (1969) 150.

[182] INCROPERA, F. P., Bower, W. W., KingSBury, R. L., Numerical Studies of Internal, Equilibrium Plasma Flows, ASME Paper 69, WA/HT, 55 (1969).

[183] Hunn, B. D., Moffat, R. J., Int. J. Heat Mass Transfer 17 (1974) 1319.

[184] Skrivan, J. F., Woldemar Von Jaskowsky, I \& E. C. Process Design and Development 4 (1965) 371.

[185] INCROPERA, F. P., I.E.E.E. Transactions on Plasma Science 3 (1973) 230.

[186] Watson, V. R., Pegot, E. B., Numerical Characteristics of a Gas Flowing axially Through a constricted Arc, NASA TN D-4042 (1967).

[187] SkIrstad, J. G., Investigation of Energy Transfer in a Tube arc Heater, A.R.L., 64-112, july (1964).

[188] Petit, A., Bonet, C., Daguenet, M., Foex, M., Etude expérimentale du transfert de chaleur entre un jet de plasma et une paroi refroidie en rotation, International Round Table on Study and application of transport phenomena in thermal plasmas, IUPAC, Odeillo, 12-16 sept. (1975) proceedings.

[189] VAN DE HulST, H. C., Light Scattering by small particles (John Wiley and Sons, New York) 1957.

[190] Starton, J. A., Electromagnetic Theory (McGraw-Hill, Book Co; New York) 1941.

[191] KeRKER, M., The scattering of light (Academic Press, London) 1969.

[192] Chu, C. M., Clark, G. G., Churchill, S. M., Tables of angular distribution coefficients for light scattering by spheries (University of Michigan Press Ann Arbor, Mich.) 1957.

[193] OzIsIK, M. N., Radiative Transfer (John Wiley and Sons, New York) 1973.

[194] De Groot, S. R., MAZUR, P., Non equilibrium thermodynamics (North Holland Publishing Co, Amsterdam) 1963.

[195] Bonet, C., Thèse de Doctorat ès Sciences, Perpignan (1973), No CNRS A.O. 8262.

[196] Gladish, H., Hydrocarbon. Process. Petrol. Refiner 41 (1962) 159.

[197] STOKes, C. S., KNIPE, W. W., StRENG, J., Electrochem. Soc. 107 (1960) 35

[198] Korman, S., Int. Sci. Tech. 30 (1964) 90.

[199] Gibson, J. O., Weidman, R., Chem. Ing. Progr. 9 (1963) 53.

[200] Holmgren, J. D., Gibson, J. O., Sheer, C., J. Electrochem. Soc. 111 (1964) 362.

[201] Kelly, A. J., J. Chem. Phys. 45 (1966) 1723.

[202] WILKs, P. H., The Survival of plasma chemistry, IUPAC 1st International Symposium on plasma chemistry Kiel Germany september (1971).

[203] Amouroux, J., Caractéristiques des sources à plasma nécessaires aux processus chimiques, Congrès National des Plasmas de la Société Française de Physique, Paris 6-10 déc. (1976).

[204] JoHN, R. R., Techniques for the Generation of Continuous High Temperature Gas Flows, The Application of Plasmas to Chemical Processing, R. G. Baddour and R. S. Timmins, editors (M.I.T. Press) 1967, pp. 13-25.

[205] ReEd, T. B., Plasmas for High Temperature Chemistry, Advances in High Temperature Chemistry, L. Eyring, editor (Academic Press) 1967, pp. 259-316.

[206] ECKERT, H. U., High Temperature Science 6 (1974) 99.

[207] BHATTACHARYA, D., Maecker effect. Theory and its application in relation to plasma technology.

[208] Bhattacharya, D., Estimation of Maecker force and its application to Industrial design of plasma arc devices.

[209] TYLKo, J. K., High Temperature Treatment of Materials, U.S. Patent 3, 783, 167. Janv. 1 (1974). 
[210] Fey, M. G., Kemeny, G. A., Method of Direct Ore Reduction Using A Short Gap Arc Heater, U.S. Patent 3, 765, 870, Oct. 16 (1973).

[211] Goldberger, W. M., Chemical Engineering Progress Symposium Series 62 (1966) 42.

[212] Bonet, C., Vallbona, G., Foex, M., Bull. Soc. Fse. Ceram. 94 (1972) 27.

[213] Bonet, C., Vallbona, G., Foex, M., Daguenet, M., DumarGUE, P., Rev. Int. Hies Temp. et Réfract. 11 (1974) 11.

[214] Whyman, D., A Rotating-wall d.c. Arc Plasma Furnace, J. Sci. Instrum. 44 (1967) 525, U.S. Patent 3, 433, 991, March 18 (1969).

[215] HowIE, F. H., SAyCE, I. G., Rev. Int. Htes Temp. et Réfract. 11 (1974) 169.

[216] SAyce, I. G., Plasma Processes in Extractive Metallurgy, Advances in Extractive Metallurgy, Institution of Mining and Metallurgy, 1972, pp. 241-274.

[217] Rykalin, N. N., Vestnik Akademic Nauk, URSS, Moscou, Russian 7 (1973) 3.

[218] Clump, W. C., Kwasnoski, D., MacRae, D. R., Thompson, C. D., A Study of High Temperature Methane Reforming, Interamerican Congress of Chemical Engineering, Caracas, Venezuela, July 13-16 (1975) pp. 33.

[219] Fifty Megawatt Electrogasdynamic Facility, Air Force Flight Dynamics Laboratory, Wright-Patterson Air Force Base, April 1967.
[220] NASA Ames Research Center, Moffett, Field, CA 94035. [221] Schoumaker, H. R. P., Document $n^{\circ} 129$ publié lors du VII' Congrès de l'U.I.E. Varsovie (1972).

[222] Bhat, G. K., J. Vac. Sci. Technol. 9 (1972) 1344.

[223] Blattman, H., Delmas, R., Foex, M., Yerouchalm D., Four rotatif de fusion, Brevet 95689 , CEA France.

[224] Aubreton, J., Dallaire, S., Yerouchalm, D., Colloque du Comité d'Electrothermie, Versailles 2-3 mai (1974).

[225] Arc Coal Process Dev. Final repport May (1966), Avril (1972), AVCO Corporation Systems Division, Lowell, Masachussetts, USA 91851.

[226] Gladisch, H., Chem. Ing. Techn. 41 (1969) 204.

[227] Soc. HoEchst AG, Hydrocarbon Proc. juin (1971).

[228] BaвсоCK, J. A., Chem. Eng. Process. 71 (1975) 90.

[229] Miller, S. A., ed. Acetylene, Its properties, Manufacture and Uses 2 (N.Y. Acad. Press) 1966.

[230] Procédé Russe, Chem. Eng. News, 24 janv. (1972) p. 38.

[231] Anderson, J. E., Case, L. K., Ind. Eng. Chem. Proc. Desg. Dev. 1 (1962) 161.

[232] Baddour, R. F., Blanchet, J. L., Ind. Eng. Chem. P.D.D. 3 (1964) 258.

[233] Meubus, P., J. Electrochem. Soc. Solid State Science and Technology 122 (1975) 298.

[234] Amouroux, dans La Chimie des Hautes Températures, ed. Chaudron, Trombe (Masson) 1974. 